\title{
Genus-one helicoids from a variational point of view
}

\author{
David Hoffman* and Brian White**
}

\begin{abstract}
In this paper, we use variational methods to prove existence of a complete, properly embedded, genus-one minimal surface that is asymptotic to a helicoid at infinity. We also prove some new properties of such helicoid-like surfaces.
\end{abstract}

Mathematics Subject Classification (2000). Primary 53A10; Secondary 49Q05.

Keywords. Complete embedded minimal surface, helicoid, variational methods.

\section{Introduction}

In this paper, we prove by variational means the existence of a complete, properly embedded, genus-one minimal surface in $\mathbb{R}^{3}$ that is asymptotic to a helicoid at infinity. We also prove existence of surfaces that are asymptotic to a helicoid away from the helicoid's axis, but that have infinitely many handles arranged periodically along the axis. These theorems were originally proved by very different methods in [WHW06]. We also prove some new properties of such helicoid-like surfaces.

To state the theorems precisely, we need some terminology. For $s \in \mathbb{R}$ we let $\sigma_{s}$ denote the screw motion of $\mathbb{R}^{3}$ defined by

$$
\sigma_{s}(\cos \theta, \sin \theta, z)=(\cos (\theta+s), \sin (\theta+s), z+s) .
$$

We let $H$ be the standard helicoid that contains the $x$-axis $X$ and the $z$-axis $Z$, and that is invariant under the screw motions $\sigma_{s}$.

A nonperiodic genus-one helicoid is a complete, properly immersed minimal surface in $\mathbb{R}^{3}$ that is conformally a once-punctured torus (i.e., a torus with one point removed) and that is asymptotic to $H$ at infinity. If it also contains $X$ and $Z$, we say that it is symmetric: by Schwarz reflection, it is invariant under the $180^{\circ}$ rotations $\rho_{X}$ and $\rho_{Z}$ about $X$ and $Z$, and hence it is also invariant under their composition $\rho_{Y}$. (Note that the symmetry group of any positive-genus surface asymptotic to $H$ must

\footnotetext{
${ }^{*}$ The research of the first author was supported by the NSF under grant DMS-0139410.

**The research of the second author was supported by the NSF under grant DMS-0406209.
} 
be a finite subgroup of the symmetry group $\operatorname{sym}(H)$ of $H$. It is not hard to prove that any finite subgroup of $\operatorname{sym}(H)$ is conjugate to a subgroup of $\left\{I, \rho_{X}, \rho_{Y}, \rho_{Z}\right\}$, and thus that a symmetric genus-one helicoid is as symmetric as a genus-one helicoid can possibly be.)

Theorem 1.1. There exists an embedded, symmetric, nonperiodic genus-one helicoid.

A periodic genus-one helicoid is a complete, properly immersed minimal surface $N$ in $\mathbb{R}^{3}$ such that for some $h>0$ :

(1) $N$ is $\sigma_{2 h}$-invariant,

(2) $N / \sigma_{2 h}$ is conformally a twice-punctured torus, and

(3) $N / \sigma_{2 h}$ is asymptotic to $H / \sigma_{2 h}$ at infinity.

If, in addition, $N$ contains $X$ and $Z$ and if the fundamental domain

$$
N \cap\{(x, y, z):|z|<h\}
$$

is bounded by the two lines $\sigma_{ \pm h}(X)$, then $N$ is called a symmetric periodic genus-one helicoid. (Note that the quotient $N / \sigma_{2} h$ of a symmetric periodic genus-one helicoid $N$ contains two horizontal lines, corresponding to the two parities of $n$ in the lines $\sigma_{n h}(X)$.)

If $N$ is any genus-one helicoid, let $h(N)$ be the smallest $h>0$ such that $N$ is $\sigma_{2 h}$-invariant. (If $N$ is nonperiodic, we let $h(N)=\infty$.)

Theorem 1.2. For every $h \in(\pi / 2, \infty]$, there is an embedded, symmetric, genus-one helicoid $N$ with $h(N)=h$.

Note that Theorem 1.1 is the special case $h=\infty$ of Theorem 1.2.

The condition $h>\pi / 2$ is sharp: in [HW], we prove that there are no examples with $h(N) \leq \pi / 2$, even if we allow somewhat less symmetry. (For $h(N)<\pi / 2$ this was observed by Meeks.)

Uniqueness is known for $h=\pi$ [WHW06], [FM05], but not in general. Thus there may be other embedded, symmetric genus-one helicoids that do not arise from the construction in this paper. The following theorem holds for all symmetric genusone helicoids:

Theorem 1.3. Let $\eta>\pi / 2$. Let $N$ be an embedded, symmetric genus-one helicoid with $h(N)=h \in[\eta, \infty]$, and let $M$ be the fundamental domain

$$
M=N \cap\{|z|<h\} .
$$


(1) The intersection $N \cap H$ consists of $Z$ together with the horizontal lines

$$
\{(x, y, z) \in H: z=n h\} \quad(n \in \mathbb{Z}) .
$$

Furthermore, $N \backslash H$ consists of congruent, simply connected components. The fundamental domain $M$ consists of two of those components, one on either side of $H$.

(2) For each vertical plane $V$, there are at most four points $p \in M \backslash Z$ for which $\operatorname{Tan}_{p} N$ is parallel to $V$. Such points must lie in the cylinder

$$
\left\{(x, y, z): x^{2}+y^{2} \leq R^{2},|z|<2 \pi\right\}
$$

where $R$ depends only on $\eta$.

(3) The space of all such $N$ (for a given $\eta>\pi / 2$ ) is compact with respect to smooth convergence on bounded subsets of $\mathbb{R}^{3}$.

If $h(N)=\infty$, then the fundamental domain $M$ is all of $N$, and the horizontal lines in assertion (1) of the theorem consist just of $X$.

Concerning assertion (1), note that by our definition of "symmetric", $N \cap H$ must contain the indicated lines. It is perhaps surprising that $N \cap H$ contains no other points. For the surfaces we produce, this property follows immediately from the construction. The proof that (1) holds in general will be given elsewhere [HW]. Proofs of (2) and (3) are given in Section 7.

A proof of assertion (3), the compactness result, by different methods is implicit in [WHW06].
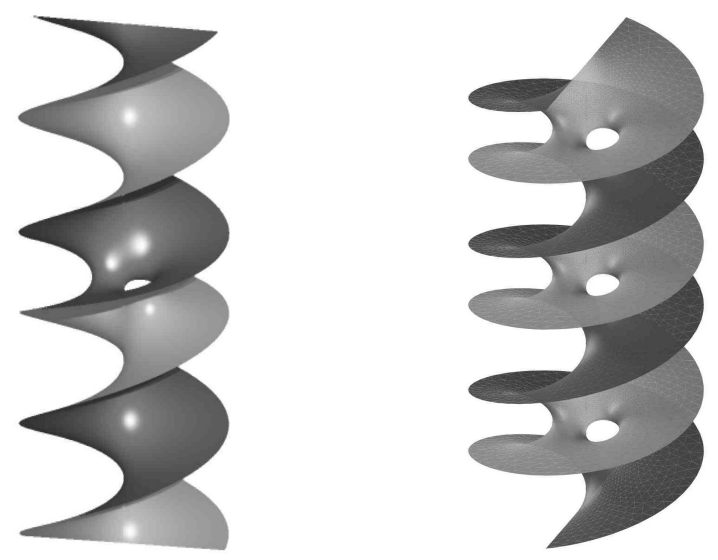

Figure 1. Nonperiodic (left) and periodic (right) genus-one helicoids.

Hoffman, Karcher, and Wei discovered symmetric genus-one helicoids. (Whether asymmetric examples exist is not known.) In [HKW93], they proved existence but 
not embeddedness of an example with $h=\infty$. In [HKW99], they proved existence of an example with $h=\pi$, and they proved that every $h=\pi$ example must be embedded. By numerical computations, they also discovered embedded examples for a range of values of $h(N)$; that work is described in [HW02].

Hoffman, Weber, and Wolf [WHW06] proved that there exists a continuous family of embedded genus-one helicoids taking all values of $h$ in $(\pi / 2, \infty]$. They also proved that there is only one example with $h=\pi$. This uniqueness was proved independently in a different way by Ferrer and Martin [FM05].

All of those investigations relied on the Weierstrass representation, which requires solution of period problems and a separate proof of embeddedness. We present here a method of realizing examples as limits of compact, embedded minimal surfaces. Period problems do not arise, and the method gives existence and embeddedness at the same time. It also provides additional information about the geometric behavior of the surfaces. Our investigation is similar in spirit to [HM88], where a variational construction of the generalized, higher-genus, Costa surfaces with three ends is given, and to the part of [CHM89] in which translation-invariant, Callahan-Hoffman-Meeks surfaces of odd genus are produced.

In our view, the construction presented here gives a good answer to the question of why genus-one helicoids should exist.

Using deep results of Colding and Minicozzi [CM04a], [CM04b], [CM04c], [CM04d], Meeks and Rosenberg proved [MR05] that the helicoid is the unique properly embedded, simply connected, nonplanar minimal surface - there is no symmetry assumption in their result. Whether or not there is only one nonperiodic, embedded, symmetric genus-one helicoid - and more generally whether there exist any nonsymmetric examples - has yet to be resolved.

The authors would like to thank Mike Wolf and Matthias Weber for helpful conversations.

\section{Outline of the construction}

2.1. The basic idea. Let $H_{R, h}$ be the portion of $H$ inside a solid cylinder centered at the origin, with axis $Z$, radius $R$, and height $2 h$ :

$$
H_{R, h}=H \cap\{|z|<h, r<R\} .
$$

The boundary $\partial H_{R, h}$ is a simple closed curve consisting of two horizontal line segments $L_{ \pm h} \subset \sigma_{ \pm h}(X)$ and two helical curves connecting the endpoints of those line segments. Note that $H_{R, h}$, like $H$, is invariant under $180^{\circ}$ rotations about the coordinate axes.

Our idea is simple: replace $H_{R, h}$ by an embedded, genus-one minimal surface $M_{R, h}$ with the same boundary. Since we want our genus-one helicoids to be symmet- 
ric, we require that $M_{R, h}$ contain the segments $X \cap H_{R, h}$ and $Z \cap H_{R, h}$. By taking a limit as $R$ and $h$ tend to infinity, we hope to get a nonperiodic genus-one helicoid. By taking a limit as $R \rightarrow \infty$ with $h$ fixed, we hope to get a fundamental domain for a $\sigma_{2 h}$-invariant periodic genus-one helicoid.

2.2. Replacing $\boldsymbol{H}_{\boldsymbol{R}, \boldsymbol{h}}$ by a genus-one surface. If $X$ and $Z$ are removed from $H_{R, h}$, four congruent "quadrants" remain. One of the quadrants contains portions of the positive rays of $X$ and $Z$ in its boundary. Another quadrant contains portions of the negative rays of $X$ and $Z$ in its boundary. We let $Q_{R, h}$ be the union of those two quadrants (See Figure 2). The rotation $\rho_{Y}$ interchanges the two quadrants, so $Q_{R, h}$
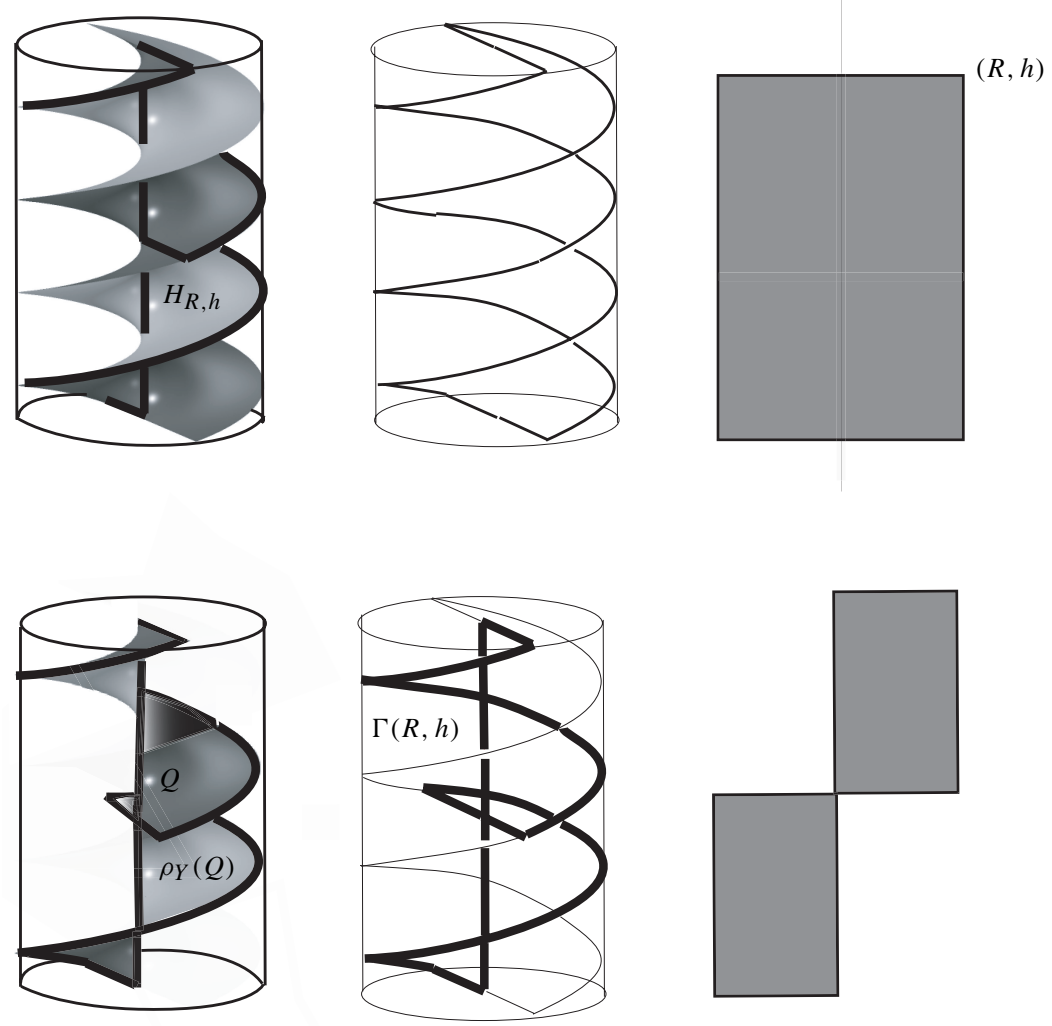

Figure 2. Top left: The surface $H_{R, h}=H \cap C$, where $C$ is the solid cylinder centered at the origin with axis $Z$, height $2 h$ and radius $R$. Top center: $\partial H_{R, h}=H \cap \partial C$. Top right: The parameter domain of $H_{R, h}$, assuming the parametrization $(u, v) \rightarrow(u \cos v, u \sin v, v)$. Bottom left: The union $Q_{R, h}$ of two of the four congruent components of $H_{R, h} \backslash(X \cup Z)$. Bottom right: The corresponding region in the parameter domain. Bottom center: $\Gamma(R, h):=\partial Q_{R, h}$. 
is $\rho_{Y}$-invariant. The boundary $\Gamma(R, h)$ of $Q_{R, h}$ is a piecewise smooth curve that is embedded except at the origin, a double point.

If in $H_{R, h}$ we replace $Q_{R, h}$ by a connected, $\rho_{Y}$-invariant minimal surface $D$ with the same boundary, then to insure $\rho_{Z}$ symmetry we must also replace $\rho_{Z}\left(Q_{R, h}\right)$ by the corresponding surface $\rho_{Z}(D)$. Because $D$ is $\rho_{Y}$-invariant, $\rho_{X}(D)=\rho_{Z}\left(\rho_{Y}(D)\right)=$ $\rho_{Z}(D)$. Thus our candidate surface is

$$
M=M_{R, h}=\bar{D} \cup \rho_{Z}(\bar{D})=\bar{D} \cup \rho_{X}(\bar{D}) .
$$

An Euler characteristic calculation shows that $M_{R, h}$ has genus one if and only if $D$ is a disk. For instance if $D$ is a disk, then we can use the nine corners and twelve edges of the quadrants of $H_{R, h}$ together with the two faces $D$ and $\rho_{Z}(D)$ to calculate that the Euler characteristic $\chi$ of $M$ is $9-12+2=-1$. Since $\partial M=\partial H_{R, h}$ has one component, its Euler characteristic and genus $g$ are related by $\chi=2-2 g-1$. Thus the genus is one, as desired. (See Figure 3.)

Note that if $D$ is embedded on one side of $H$, then $\rho_{Z}(D)$ will lie on the other side of $H$ and thus $M$ will be embedded. By Schwarz reflection (see (1)), the interior of $M$ will be smoothly embedded along $X$ and $Z$ except possibly at the origin. Since an embedded minimal surface of finite topology in $\mathbb{R}^{3}$ cannot have an isolated interior singularity ([Nit89], §363), in fact the interior of $M$ is smoothly embedded everywhere.
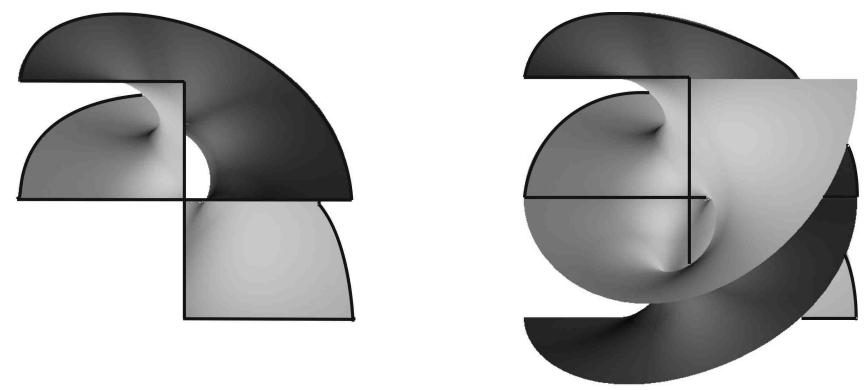

Figure 3. Left: An embedded, $\rho_{Y}$-invariant minimal disk $D$ on one side of $H$ with $\partial D=$ $\Gamma(R, h)$. In this illustration, $h=\pi$. Right: The genus-one surface $M=\bar{D} \cup \rho_{Z}(\bar{D})$.

We can extend $M_{R, h}$ to get a $\sigma_{2 h}$-invariant surface

$$
N=N_{R, h}=\bigcup_{n \in \mathbb{Z}} \sigma_{2 h n}\left(M_{R, h}\right)
$$

This surface is clearly embedded, and its boundary consists of two helices. Since we can also obtain $N$ from $M$ by repeated Schwarz reflections about the top and bottom edges, the interior of $N$ will be smooth (as was the case with $M$.) Since $N$ 
has locally finite topology and since $\partial N$ is smooth and lies on the boundary of the convex hull of $N$, there are no boundary singularities ([Nit89], §366). That is, $N$ is smooth everywhere.

To summarize our discussion, we have the following procedure for generating symmetric genus-one helicoids:

- Find a $\rho_{Y}$-invariant minimal embedded disk $D=D_{R, h}$ that has boundary $\Gamma(R, h)$ and that lies on one side of $H$.

- Form the corresponding smooth embedded genus-one surface $M=M_{R, h}$ and the corresponding smooth, embedded $\sigma_{2 h}$-invariant surface $N=N_{R, h}$.

- Take a limit of $M_{R, h}$ (or, equivalently, of $N_{R, h}$ ) as $R$ and $h$ tend to infinity to get a nonperiodic example. Fix $h$ and take a limit of $N_{R, h}$ as $R \rightarrow \infty$ to get a periodic, $\sigma_{2 h}$-invariant example.

Here and throughout the paper, "disk" means "open disk". (Since $\Gamma(R, h)$ has a double point, $\bar{D}$ will not be embedded even if $D$ is embedded.) To avoid tedious repetition, we will let "disk" mean "embedded, $\rho_{Y}$-invariant disk" for the remainder of this section.

2.3. What could go wrong? To prove that the procedure we have just described works, we must address the following questions:

(1) How do we know that there is a minimal disk $D$ on one side of $H$ with $\partial D=$ $\Gamma(R, h)$ ?

(2) How do we know that we have smooth convergence as $R$ tends to infinity, or as $R$ and $h$ both tend to infinity?

(3) How do we know that the limit surface has the desired topology?

Concerning question (1), there is no such minimal disk when $R$ is very small or when $h \leq \pi / 2$. Indeed, in those cases one can prove that $Q_{R, h}$ is the unique minimal variety with boundary $\Gamma(R, h)$, and it is not a disk, but rather two disks. Fortunately, we need minimal disks $D$ only for $h>\pi / 2$ and $R$ very large.

We will discuss the curvature estimates that address question (2) later. The key to questions (1) and (3) turns out to be the following fact:

Proposition 2.4. Let $h>\pi / 2$. For all sufficiently large $R$, there exists a minimal annulus $A$ that has boundary in $Q_{R, h}$ and that lies in the component $H^{+}$of $\mathbb{R}^{3} \backslash H$ containing the positive $y$-axis.

Sketch of proof. (See Proposition 3.3 for details.) Note that when $R$ is very large, the region $H^{+}$near the point $(0, R / 2,0)$ resembles a slab between two horizontal planes. Consequently, the intersection of $H^{+}$with a suitable catenoid centered at $(0, R / 2,0)$ has one component that is an annulus $A$ with boundary in $H$. The condition $h>\pi / 2$ 
is precisely the condition that guarantees (provided $R$ is sufficiently large) that $\partial A$ lies in $Q_{R, h}$. (Figure 4 shows the case $h=\pi$.)

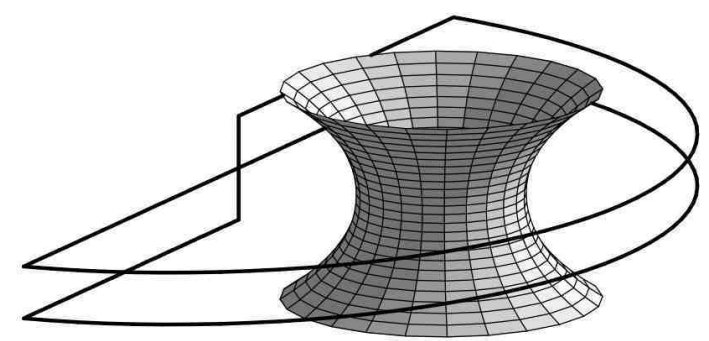

Figure 4. The curve $\Gamma=\Gamma(R, \pi)$ and a catenoidal barrier. A minimal disk that has boundary $\Gamma$ and that avoids the barrier must lie close to $\Gamma$, because if we translate the barrier horizontally, it must touch $\Gamma$ before it touches the interior of the disk.

We can now get a minimal disk in $H^{+}$by minimizing area among disks $D$ that have boundary $\Gamma(R, h)$ and that lie in the unbounded component of $H^{+} \backslash A$.

(Since the standard theorems about minimal disks assume embedded boundary, we should first approximate $\Gamma(R, h)$ by embedded smooth curves in $H$, but in this outline we will ignore such technicalities.)

However, that least-area disk $D^{*}$ is not the one we want. Consider for example the case $h=\pi$. By construction, the disk $D^{*}$ is disjoint from some catenoid passing through the middle of $\Gamma(R, h)$, as shown in Figure 4. By translating the catenoid around horizontally, we see from the maximum principle that $D^{*}$ is forced to lie close to its boundary. Consequently, if we take a limit of such $D^{*}$ as $R \rightarrow \infty$, then we are left just with two flat strips in the $x z$-plane. The corresponding complete embedded surface (generated by Schwarz reflections from those strips) is the $x z$-plane, not the periodic genus-one helicoid we want.

The problem is a loss of topology. The closure of $D^{*}$ is not simply connected: it has a closed geodesic starting and ending at the origin. But as $R \rightarrow \infty$, the length of that geodesic tends to infinity. Thus the limiting geodesic is not closed, and the limiting surface is simply connected.

Similar reasoning shows that in general our procedure is doomed to yield a simply connected limit surface unless we use minimal disks $D$ with $\partial D=\Gamma(R, h)$ that have the following annular intersection property: $D$ intersects every minimal annulus $A$ in $H^{+}$with $\partial A \subset Q_{R, h}$.

For minimal disks $D$ that have the annular intersection property, we prove a uniform bound on the length of the geodesic starting and ending at the origin. This implies that a limit of such disks will generate a surface with the desired topology. (In 
particular, the surface will contain a closed geodesic and therefore will have nontrivial fundamental group.)

Thus our scheme for producing genus-one helicoids works if and only if we use disks that have the annular intersection property. Fortunately such disks do exist:

Proposition 2.5. Let $h>\pi / 2$. For all sufficiently large $R$, the curve $\Gamma(R, h)$ bounds a $\rho_{Y}$-invariant minimal embedded disk in $H^{+}$that has the annular intersection property.

Sketch of proof. (See Section 3 for details.) By Proposition 2.4 and the sentence following its proof, the set $\mathcal{F}$ of minimal disks in $H^{+}$bounded by $\Gamma(R, h)$ is nonempty. For simplicity, let us assume that $\mathcal{F}$ is a finite set, and that each disk in $\mathcal{F}$ is strictly stable or strictly unstable. Choose a disk $D \in \mathcal{F}$ that is closest to $Q_{R, h}$ in the sense that no other disk in $\mathcal{F}$ lies between $D$ and $Q_{R, h}$. We will show that $D$ must have the annular intersection property.

Note that $Q_{R, h}$ is strictly stable: each of its two components lies in a half-helicoid (i.e., one of the components of $H \backslash Z$ ), and each half-helicoid is stable because its Gauss-map image lies in a hemisphere.

If $D$ were strictly stable, then (by a general minimax or mountain pass lemma) there would be an unstable disk between $D$ and $Q_{R, h}$, contradicting the choice of $D$. Thus $D$ is strictly unstable.

Now consider a minimal annulus $A$ in $H^{+}$with $\partial A \subset Q_{R, h}$. If $A$ were disjoint from $D$, we could minimize area among all disks that have boundary $\Gamma(R, h)$ and that lie in the region of $H^{+}$between $D$ and $A$. By the instability of $D$, the result would be a minimal disk $D^{\prime}$ lying strictly between $D$ and $Q_{R, h}$, contradicting the choice of $D$. Thus $D$ intersects every such annulus. That is, $D$ has the annular intersection property.

We end this outline by saying a word about the curvature estimates that guarantee smooth convergence in question (2) of Section 2.3 above. The points of a disk $D \subset \mathbb{R}^{3}$ with vertical tangent planes are of course the critical points of nonzero linear functions of the form $f(x, y, z)=a x+b y$. Morse theory lets us deduce facts about the set of such critical points from knowledge of the boundary. In that way, in Section 4, we control the set of points in $D$ with vertical tangent planes. That control in turn lets us deduce curvature estimates in Section 5.

\section{Disks with the annular intersection property}

3.1. Let $H_{R, h}$ be the intersection of the helicoid $H$ with a solid right-circular cylinder centered at the origin with axis $Z$, radius $R$, and height $2 h$. Thus

$$
H_{R, h}=F((-R, R) \times(-h, h))
$$


where

$$
F(u, v)=(u \cos v, u \sin v, v) .
$$

The $x$ - and $z$-axes divide $H_{R, h}$ into four congruent "quadrants". Let $Q_{R, h}$ denote the union of the first and third quadrants:

$$
Q_{R, h}=F((0, R) \times(0, h)) \cup F((-R, 0) \times(-h, 0)) .
$$

Note that $Q_{R, h}$ consists of two pieces that have a common corner at the origin and that are related by the $180^{\circ}$ rotation $\rho_{Y}$ about the $y$-axis, $Y$. (See Figure 2.)

We let $\Gamma(R, h)$ be the boundary of $Q_{R, h}$. We will regard $\Gamma(R, h)$ as a piecewise smooth curve that is embedded except at the origin, a double point. Note that $\Gamma(R, h)$ consists of line segments together with the two helical arcs

$$
\{F(R, v): 0 \leq v \leq h\} \quad \text { and } \quad\{F(-R, v):-h \leq v \leq 0\} .
$$

Recall that $H^{+}$is the component of $\mathbb{R}^{3} \backslash H$ that contains the positive $y$-axis. Our goal in this section is to prove existence of a $\rho_{Y}$-invariant minimal disk in $H^{+}$with boundary $\Gamma(R, h)$ and with the following "annular intersection property":

Definition 3.2. If $\Gamma$ is closed curve in $H$, let $\mathcal{A}(\Gamma)$ be the set of minimal embedded annuli $A$ in $H^{+}$such that $\partial A$ is smooth and is contained in the union of the bounded components of $H \backslash \Gamma$. A minimal surface with boundary $\Gamma$ that intersects every annulus in $\mathcal{A}(\Gamma)$ is said to have the annular intersection property.

Of course the annular intersection property is vacuous if $A(\Gamma)$ is empty. However, $\mathcal{A}(\Gamma(R, h))$ is nonempty for suitable $R$ and $h$ :

Proposition 3.3. For every $\eta>\pi / 2$, there is an $R_{\eta}<\infty$ such that $\mathcal{A}(\Gamma(R, h))$ is nonempty provided $R \geq R_{\eta}$ and $h \geq \eta$.

Proof. Note that for $h \geq \eta$,

$$
Q(R, \eta) \subset Q(R, h)
$$

and so $\mathcal{A}(\Gamma(R, \eta)) \subset \mathcal{A}(\Gamma(R, h))$. Thus it suffices to prove that $\mathcal{A}(R, \eta)$ is nonempty for all sufficiently large $R$. For the same reason, it suffices to consider $\eta$ with $\pi / 2<$ $\eta \leq \pi$.

Translate $H^{+}$and $Q_{R, \eta}$ by $(0,-R / 2,0)$ to obtain $\left(H^{+}\right)_{R}^{\prime}$ and $Q_{R, \eta}^{\prime}$. Note that as $R \rightarrow \infty,\left(H^{+}\right)_{R}^{\prime}$ converges to a limit $\left(H^{+}\right)^{\prime}$ consisting of horizontal slabs, one of which is the slab $|z| \leq \pi / 2$. Also, $Q_{R, \eta}^{\prime}$ converges smoothly to the union $Q^{\prime}$ of the planes $z=\pi / 2$ and $z=-\pi / 2$.

Let $C$ be any catenoid with a vertical axis of symmetry. Note that $C$ intersects the planes $z= \pm \pi / 2$ transversely in a pair of circles that bound an annular portion 
of $C$ in $\left(H^{+}\right)^{\prime}$. Hence for all sufficiently large $R$, the catenoid $C$ intersects $Q_{R, \eta}^{\prime}$ in a pair of curves that bound an annular component $A_{R}$ of $C$ in $\left(H^{+}\right)_{R}$. Translating $A_{R}$ by $(0, R / 2,0)$ produces a minimal annulus in $H^{+}$with boundary in $Q_{R, \eta}$.

3.4. The existence result for smooth simple closed curves $\Gamma$. The closed curve $\Gamma(R, h)$ is neither simple nor smooth. We first prove the result we desire for smooth simple closed curves $\Gamma$ that approximate $\Gamma(R, h)$. We then prove the estimates (Lemma 3.9) that allow us conclude the desired result (Theorem 3.11) for $\Gamma(R, h)$.

Theorem 3.5. Suppose $\Gamma$ is a smooth simple closed curve in $H$ such that

(1) the region $D_{\Gamma}$ in $H$ bounded by $\Gamma$ is strictly stable, and

(2) $\mathcal{A}(\Gamma)$ is nonempty.

Then $\Gamma$ bounds a weakly unstable minimal embedded disk $D$ in $\mathrm{H}^{+}$with the annular intersection property.

If $\Gamma$ is $\rho_{Y}$-invariant, then we may require that $D$ also be $\rho_{Y}$-invariant.

Proof. Consider first the case that $\Gamma$ is noncritical in the following sense: 0 is not an eigenvalue of the Jacobi operator of any smooth embedded minimal disk in $\mathrm{H}^{+}$with boundary $\Gamma$.

Let $\mathcal{F}=\mathcal{F}(\Gamma)$ be the set of minimal embedded disks in $H^{+}$bounded by $\Gamma$. Then $\mathcal{F} \cup\left\{D_{\Gamma}\right\}$ is compact by standard curvature estimates (see for example [Whi87b] or Lemma 3.9 below.) Moreover, since $D_{\Gamma}$ is strictly stable and since $\Gamma$ is noncritical, $\mathcal{F}$ is in fact finite.

Let $A$ be an annulus in $\mathcal{A}(\Gamma)$. We claim that $\Gamma$ bounds an embedded disk in $H^{+}$ disjoint from $A$. To see this, let $\mathbb{B}$ be a large ball in $\mathbb{R}^{3}$ centered at the origin with $\Gamma$ in its interior. Note that $H \cap \mathbb{B}$ and $H^{+} \cap(\partial \mathbb{B})$ are topologically disks with a common boundary, so their union $S$ is topologically a sphere. Thus $\Gamma$ divides $S$ into two regions, $D_{\Gamma}$ and $S \backslash D_{\Gamma}$, each of which is topologically a disk. In particular, $S \backslash D_{\Gamma}$ is a piecewise smooth embedded disk in $\overline{H^{+}}$that has boundary $\Gamma$ and that is disjoint from $A$. By perturbing slightly, we get a smooth embedded disk in $H^{+} \backslash A$ with boundary $\Gamma$.

Now minimize area among all disks in $H^{+} \backslash A$ with boundary $\Gamma$. The minimum exists and is smoothly embedded by a theorem of Meeks and Yau (see Theorem 3.6 below), and thus it is a disk in the family $\mathcal{F}$. By finiteness of $\mathcal{F}$, there is a disk $D \in \mathcal{F}$ that is closest to $D_{\Gamma}$ in the sense that no other disk in $\mathcal{F}$ lies between $D$ and $D_{\Gamma}$.

Now if $D$ were stable, it would be strictly stable by noncriticality of $\Gamma$. But then by a standard minimax principle (see for example Theorem A.1 in the appendix), there would be an unstable minimal embedded disk between $D$ and $D_{\Gamma}$, contradicting the choice of $D$. Thus $D$ is strictly unstable. 
It remains only to show that $D$ must intersect every annulus in $\mathcal{A}(\Gamma)$. Suppose on the contrary that $\mathcal{A}(\Gamma)$ contains an annulus $A$ disjoint from $D$. Let $D^{\prime}$ be the least-area embedded disk bounded by $\Gamma$ in the closure of the region of $\mathrm{H}^{+}$between $A$ and $D$. (The disk exists and is smoothly embedded by the Meeks-Yau theorem.) Since $D_{\Gamma}$ does not lie in the closure of that region, $D^{\prime} \neq D_{\Gamma}$. Since $D$ is strictly unstable, $D^{\prime} \neq D$. Thus $D^{\prime}$ lies between $D$ and $D_{\Gamma}$, contradicting the choice of $D$. Hence $D$ intersects $A$ as claimed.

This completes the proof in the case of noncritical $\Gamma$. In fact, noncritical $\Gamma$ are generic (see Theorem A.2 in the appendix). Thus we can find a nested sequence of noncritical $\Gamma_{i} \subset H$ converging smoothly to $\Gamma$ from the outside. Let $D_{i}$ be an unstable embedded disk in $H^{+}$bounded by $\Gamma_{i}$ and intersecting all the $A \in \mathcal{A}\left(\Gamma_{i}\right)$. Note that $\mathcal{A}(\Gamma) \subset \mathcal{A}\left(\Gamma_{i}\right)$ since $\Gamma_{i}$ encloses $\Gamma$ in $H$. Thus $D_{i}$ intersects every $A \in \mathcal{A}(\Gamma)$. The curvatures of the $D_{i}$ are bounded by standard estimates (see [Whi87b] or Lemma 3.9 below.) Thus a subsequence converges smoothly to a limit disk $D$ in $\overline{H^{+}}$. Now $D$ is weakly unstable (since it is the smooth limit of unstable disks), so $D \neq D_{\Gamma}$. Hence (by the strong maximum principle) $D$ cannot touch $H$, so $D$ lies in $H^{+}$. Also, $D$ intersects every $A \in \mathcal{A}(\Gamma)$ since each $D_{i}$ does. This completes the proof for arbitrary $\Gamma$.

Finally, the proof in case of $\rho_{Y}$-invariance is exactly the same, except that we work throughout with $\rho_{Y}$-invariant curves and disks, and with the restriction of the Jacobi operator to the space of $\rho_{Y}$-equivariant vectorfields. The minimal annulus $A$ should be replaced by $A \cup \rho_{Y}(A)$. Where the proof uses a least-area disk $\Delta$, that disk turns out to be $\rho_{Y}$-invariant. For if $\Delta$ were not $\rho_{Y}$-invariant, then $\rho_{Y}(\Delta)$ would be a second least-area disk with the same boundary. By the Meeks-Yau theorem, $\Delta$ and $\rho_{Y}(\Delta)$ would be disjoint. But by the $\rho_{Y}$ symmetry, the volume of the region between $D_{\Gamma}$ and $\Delta$ must equal the volume of the region between $D_{\Gamma}$ and $\rho_{Y}(\Delta)$, so $\Delta$ and $\rho_{Y}(\Delta)$ cannot be disjoint. The contradiction proves that $\Delta$ is indeed $\rho_{Y}$-invariant.

For the reader's convenience, we state the theorem of Meeks and Yau that was used in the preceding proof:

Theorem 3.6 (Meeks-Yau, [MY82a], [MY82b]). Let $\Omega \subset \mathbb{R}^{3}$ be a mean convex domain with piecewise smooth boundary, and let $\Gamma$ be a smooth curve in $\partial \Omega$ that bounds a disk in $\Omega$. Then $\Gamma$ bounds a least-area disk $D$ in $\bar{\Omega}$. Such a disk must be smooth and embedded, and it must be contained either in $\partial \Omega$ or else in $\Omega$. Furthermore, any two such disks must be disjoint.

Meeks and Yau prove this theorem for convex domains in Theorem 6 of [MY82a]. They extend the result to other domains in Section 1 of [MY82b].

3.7. Approximation results and uniform curvature estimates. Note that a halfhelicoid (such as either component of $H \backslash Z$ ) is stable since its image under the 
Gauss map is contained in a hemisphere. Consequently any bounded domain in a half-helicoid is strictly stable. In particular, the union $Q=Q_{R, h}$ of the bounded components of $H \backslash \Gamma(R, h)$ is strictly stable. In the following lemma, we show that $Q$ can be fattened to get a strictly stable domain $\Omega \subset H$ bounded by a smooth simple closed curve.

Lemma 3.8. For any positive numbers $R$ and $h$, there is a simply connected, $\rho_{Y}$-invariant domain $\Omega \subset H$ that contains $\overline{Q_{R, h}}$, is strictly stable, and is bounded by a smooth embedded curve.

Proof. Let $\Omega_{i}$ be the set of points in $H$ at distance $\leq 1 / i$ from the union $Q=Q_{R, h}$ of the two bounded components of $H \backslash \Gamma$. Let $\lambda_{i}$ and $\lambda$ be the lowest eigenvalues of the Jacobi operator on $\Omega_{i}$ and on $Q$, respectively. Let $u_{i}$ be the first eigenfuction on $\Omega_{i}$, normalized so that $\max u_{i}=1$. Recall that $u_{i}>0$ at every interior point. Note that

$$
\lambda_{1}<\lambda_{2}<\lambda_{3}<\cdots
$$

and that $\lambda_{i}<\lambda$ for all $i$, so that

$$
\lambda_{\infty} \leq \lambda
$$

where

$$
\lambda_{\infty}=\lim _{i} \lambda_{i} .
$$

By the Schauder estimates, we may assume that the $u_{i}$ converge to a limit $u$, the convergence being smooth away from the corners of $\Gamma$.

(If the smooth convergence is not clear, note that the $u_{i}$ are uniformly bounded since they are normalized to have maximum value 1 . Thus the Schauder estimates give uniform local $C^{2, \alpha}$ bounds as $i \rightarrow \infty$ away from the corners of $\Gamma$. Those bounds imply $C^{2}$ convergence away from the corners for a subsequence of the $u_{i}$. Likewise, higher-order Schauder estimates imply $C^{k}$ convergence for each $k$.)

Claim. The convergence $u_{i} \rightarrow u$ is uniform up to and including the boundary.

Proof of claim. It suffices to show the following: if $p_{i} \in \Omega_{i}$ converges to $p \in \Gamma$, then $u_{i}\left(p_{i}\right)$ converges to 0 . To see that it does, fix a $p \in \Gamma$ and let $a$ be the supremum of

$$
\lim \sup u_{i}\left(p_{i}\right)
$$

among all sequences $p_{i} \in \Omega_{i}$ with $p_{i} \rightarrow p$. Note that the supremum is attained by some sequence $p_{i}$. By passing to a subsequence, we may assume that

$$
\lim u_{i}\left(p_{i}\right)=a .
$$

Our goal is to show that $a=0$. 
Translate $\Omega_{i}$ by $-p_{i}$ and dilate by

$$
\mu_{i}:=\frac{1}{\operatorname{dist}\left(p_{i}, \partial \Omega_{i}\right)}
$$

to get a surface $\Omega_{i}^{\prime}$. Note that

$$
\mu_{i} \rightarrow \infty
$$

By passing to a subsequence, we can assume that the $\Omega_{i}^{\prime}$ converge to a plane domain $\Omega^{\prime}$ with

$$
\operatorname{dist}\left(0, \partial \Omega^{\prime}\right)=1
$$

so that in particular $\partial \Omega^{\prime}$ is not empty. Note that $\partial \Omega^{\prime}$ is either smooth or piecewise smooth. (Indeed, $\partial \Omega^{\prime}$ must be a straight line, or two rays together with a quarter circle joining their endpoints, or a right angle, or the union of two disjoint right angles. Here "right angle" means "union of two orthogonal rays with a common endpoint".)

Let $u_{i}^{\prime}$ be the function on $\Omega_{i}^{\prime}$ corresponding to $u_{i}$. Then $u_{i}^{\prime}$ is a Jacobi eigenfuction with eigenvalue

$$
\lambda_{i}^{\prime}=\frac{\lambda_{i}}{\mu_{i}^{2}}
$$

Note that $\lambda_{i}^{\prime} \rightarrow 0$ (because by (4) the $\mu_{i}$ tend to infinity and by (3) the $\lambda_{i}$ 's are bounded.) Thus by passing to a subsequence we may suppose that the $u_{i}^{\prime}$ converge smoothly away from the corners of $\partial \Omega^{\prime}$ to a Jacobi eigenfunction

$$
u^{\prime}: \Omega^{\prime} \rightarrow \mathbb{R}
$$

with eigenvalue 0 . Since $\Omega^{\prime}$ is planar, $u^{\prime}$ is in fact a harmonic function. Note that

$$
\max u^{\prime}=u^{\prime}(0)=a .
$$

Thus by the maximum principle for harmonic functions, $u^{\prime}$ is constant. But $u^{\prime}$ vanishes on the smooth portions of $\partial \Omega^{\prime}$, so $a$ must be 0 . This completes the proof of the claim.

We now resume the proof of the lemma. By the uniform convergence $u_{i} \rightarrow u, u$ is nonzero (its maximum value is 1 ) and it vanishes at the boundary. By the smooth convergence on the interior, it is a Jacobi eigenfunction on $Q$ with eigenvalue $\lambda_{\infty}$. Since $Q$ is strictly stable (see the discussion immediately preceding the lemma), $\lambda_{\infty}>0$.

Thus there is an $n$ (any sufficiently large $n$ will do) for which $\Omega_{n}$ is strictly stable. Now let $\Omega$ be any $\rho_{Y}$-invariant domain in $H$ such that

$$
\bar{Q} \subset \Omega \subset \Omega_{n}
$$

and such that $\partial \Omega$ is a smooth simple closed curve. 
The next lemma follows from the estimates in [Whi87b], but since the proof is short, we include it for the reader's convenience.

Lemma 3.9. Let $D_{i}$ be a sequence of embedded minimal disks in $\mathrm{H}^{+}$with embedded, piecewise smooth boundary curves $\Gamma_{i}$ in $H$. Suppose that the $\Gamma_{i}$ converge to a curve $\Gamma$ that is smooth and embedded except at a finite set $S$, and suppose that the convergence $\Gamma_{i} \rightarrow \Gamma$ is smooth on compact subsets of $\mathbb{R}^{3} \backslash S$. Suppose also that the total curvatures of the $\Gamma_{i}$ are uniformly bounded.

Then the principal curvatures of the $D_{i}$ are uniformly bounded as $i \rightarrow \infty$ on compact subsets of $\mathbb{R}^{3} \backslash S$.

Proof. Suppose the lemma fails. Then (by passing to a subsequence) there is a compact set $K$ disjoint from $S$ and a sequence $p_{i} \in K \cap \bar{D}_{i}$ such that

$$
B\left(\overline{D_{i}}, p_{i}\right) \rightarrow \infty .
$$

Here $B\left(\overline{D_{i}}, p_{i}\right)$ is the norm of the second fundamental form of $\overline{D_{i}}$ at $p_{i}$. By enlarging $K$ slightly, we can assume that

$$
B\left(\overline{D_{i}}, p_{i}\right) \operatorname{dist}\left(p_{i}, \partial K\right) \rightarrow \infty .
$$

Fixing this $K$, we may rechoose $p_{i} \in K \cap \overline{D_{i}}$ to maximize the left hand side of (5).

Now translate $D_{i}, K$, and $H$ by $-p_{i}$ and dilate by

$$
\mu_{i}:=B\left(\overline{D_{i}}, p_{i}\right)
$$

to get $D_{i}^{\prime}, K_{i}^{\prime}$, and $H_{i}^{\prime}$ such that

$$
B\left(\bar{D}_{i}^{\prime}, 0\right)=1
$$

and such that

$$
\max _{p \in K_{i}^{\prime} \cap \bar{D}_{i}^{\prime}} B\left(\bar{D}_{i}^{\prime}, p\right) \operatorname{dist}\left(p, \partial K_{i}^{\prime}\right)=\operatorname{dist}\left(0, \partial K_{i}^{\prime}\right) \rightarrow \infty .
$$

From this we see that the $\partial K_{i}^{\prime}$ are moving off to infinity and that the curvatures of the $D_{i}^{\prime}$ are uniformly bounded as $i \rightarrow \infty$ on compact subsets of $\mathbb{R}^{3}$. Note that $\partial D_{i}^{\prime}$ converges either to the empty set (i.e., it moves off to infinity) or to a straight line, the convergence in the latter case being smooth on compact subsets of $\mathbb{R}^{3}$. Thus we may assume that the $D_{i}^{\prime}$ converge smoothly to a limit minimal surface $D^{\prime}$. After passing to a subsequence, the $H_{i}^{\prime}$ will converge smoothly to a limit $H^{\prime}$ that is either a plane or the empty set.

Now $D^{\prime}$ is simply connected and embedded, and

$$
B\left(D^{\prime}, 0\right)=1
$$


by (6). By the Gauss-Bonnet theorem, the total curvatures of the $D_{i}$ are uniformly bounded and thus $D^{\prime}$ has finite total curvature.

If $D^{\prime}$ has no boundary, then it is a complete, embedded, simply connected minimal surface of finite total curvature. But the only such surface is a plane, contradicting (7).

Thus $D^{\prime}$ has nonempty boundary, namely a line. Note that the line lies in a plane, namely $H^{\prime}$, and that $D^{\prime}$ lies in a closed halfspace bounded by $H^{\prime}$. Thus extending $D^{\prime}$ by Schwarz reflection produces a complete, simply connected, embedded minimal surface of finite total curvature. Again, the only such surface is a plane, contradicting (7).

3.10. The existence result for $\Gamma(\boldsymbol{R}, \boldsymbol{h})$. Recall that $\mathcal{A}(\Gamma)$ is the set of minimal annuli $A$ in $H^{+}$with $\partial A$ contained in the union of the bounded components of $H \backslash \Gamma$, and that $\Gamma(R, h)$ is the piecewise smooth curve defined in Section 3.1.

Theorem 3.11. Let $\Gamma=\Gamma(R, h)$ and suppose $\mathcal{A}(\Gamma)$ is not empty. Then $\Gamma$ bounds a $\rho_{Y}$-invariant minimal disk $D$ in $H^{+}$such that

(1) $\bar{D}$ is smoothly embedded except at the corners of $\Gamma$, and

(2) D has the annular intersection property (Definition 3.2).

Proof. By Lemma 3.8, $H$ contains a $\rho_{Y}$-invariant, simply connected, strictly stable domain $\Omega$ containing $\Gamma$ such that $\partial \Omega$ is a smooth embedded curve.

Now let $\Gamma_{i}$ be a sequence of smooth $\rho_{Y}$-invariant simple closed curves in $\Omega$ such that

(1) $\Gamma_{i}$ encloses $\Gamma$,

(2) $\Gamma_{i}$ converges to $\Gamma$ as $i \rightarrow \infty$, the convergence being smooth away from the corners of $\Gamma$, and

(3) the total curvatures of the $\Gamma_{i}$ are uniformly bounded.

Since the region of $H$ bounded by $\Gamma_{i}$ is a subset of $\Omega$, it must be strictly stable. Since $\Gamma_{i}$ encloses $\Gamma, \mathcal{A}\left(\Gamma_{i}\right)$ contains $\mathcal{A}(\Gamma)$ and is therefore nonempty.

Thus by Theorem 3.5, $\Gamma_{i}$ bounds a $\rho_{Y}$-invariant minimal embedded disk $D_{i}$ in $H^{+}$ that intersects every annulus $A$ in $\mathcal{A}\left(\Gamma_{i}\right)$. In particular, $D_{i}$ intersects every annulus $A$ in $\mathcal{A}(\Gamma)$.

By Lemma 3.9, the curvatures of the $D_{i}$ are uniformly bounded away from the corners of $\Gamma$. Thus by passing to a subsequence we may assume that the $D_{i}$ converge smoothly (away from those corners) to an embedded minimal surface $D$ in the closure of $H^{+}$with $\partial D=\Gamma$.

We claim that $D$ is not contained in $H$. To see that this is the case, note that the strict stability of $\Omega$ implies that $\Omega$ is contained in an open set $W$ of $\mathbb{R}^{3}$ with the following property: any minimal surface with boundary in $\Omega$ either is entirely contained in $\Omega$ or else contains points in $W^{c}$. (See Corollary A.4 in the appendix.) 
Since $\partial D_{i}$ is contained in $\Omega$ and $D_{i}$ is not contained in $\Omega$, we see that $D_{i} \cap W^{c}$ is not empty. Thus $D \cap W^{c}$ is not empty, which implies that $D$ is not contained in $H$, as claimed.

Since the $D_{i}$ are simply connected, each component of $D$ is simply connected. We claim that $D$ consists of only one connected component. To see that it does, note that $\Gamma$ may be regarded as the union of two simple closed curves $C^{\prime}$ and $C^{\prime \prime}$ that intersect at the origin. Suppose that $D$ is not connected. Then it must consist of two components $D^{\prime}$ and $D^{\prime \prime}$ with boundary curves $C^{\prime}$ and $C^{\prime \prime}$, respectively. Note that $D^{\prime \prime}=\rho_{Y}\left(D^{\prime}\right)$ by the $\rho_{Y}$ symmetry of $D$. Since $D$ does not lie in $H$, neither $D^{\prime}$ nor $D^{\prime \prime}$ can lie in $H$. Note that $C^{\prime}$ lies in the closure $\Sigma^{\prime}$ of one of the two connected component of $H \backslash Z$ : it consists of a segment $I$ of the $z$-axis, two horizonal segments, and a helical curve. Now rotate the other half-helicoid $\Sigma^{\prime \prime}=H \backslash \Sigma^{\prime}$ about the $z$-axis in $H^{+}$until it first either touches $D^{\prime}$ on the interior or becomes tangent to $D^{\prime}$ along the $z$-axis. It cannot touch at an interior point by the maximum principle. It cannot touch at the endpoints of $I$ since $D^{\prime}$ is tangent to $H$ there. Thus the first point of contact is a point of tangency at a point inside the segment $I$. But that violates the boundary maximum principle. This contradiction proves that $D$ is connected.

Since $D$ lies in the closure of $H^{+}$but does not lie in $H$, by the strict maximum principle $D$ cannot touch $H$ at any interior point. That is, $D$ is contained in $\mathrm{H}^{+}$.

Finally, $D$ intersects every $A \in \mathcal{A}(\Gamma)$ since each $D_{i}$ does.

\section{Vertical tangent planes}

Throughout this section, we will assume that $R$ and $h$ are fixed with $h>\pi / 2$, and that $D \subset H^{+}$is an embedded, $\rho_{Y}$-invariant minimal disk with boundary $\partial D=\Gamma=$ $\Gamma(R, h)$, the curve specified in Section 3.1. As explained in Section 2.2,

$$
M:=\bar{D} \cup \rho_{Z}(\bar{D})=\bar{D} \cup \rho_{X}(\bar{D})
$$

is a smooth, embedded genus-one minimal surface, and

$$
N:=\bigcup_{n \in \mathbb{Z}} \sigma_{2 n h}(M)
$$

is a smoothly embedded, $\rho_{2 h}$-invariant minimal surface whose boundary is a pair of helices.

We will prove in Proposition 4.4 that there are at most two interior points of $D$ at which the tangent plane is parallel to a given vertical plane. Furthermore, such points must be close (within distance $2 \pi$ ) to the $x y$-plane. We will give similar bounds on the number of times a vertical plane $V$ can be tangent to $D$ along $Z$ (Theorem 4.10). We combine these results to get a local upper bound (Theorem 4.13) on the number of points in $N$ where the tangent plane is parallel to a given vertical plane $V$. 
The theorems of this section, all of which control the set of points with vertical tangent planes, will be used in Section 5 to get the curvature estimates needed when we let $R$ and/or $h$ tend to infinity.

A key ingredient in the proof of Proposition 4.4 is a generalization of Rado's theorem, according to which the tangent plane to a minimal disk in $\mathbb{R}^{3}$ must intersect the boundary in at least four points. Rado's theorem has the following consequence: if the restriction of a linear function $f$ to the boundary of a minimal disk has only one local maximum, then $f$ has no interior critical points in the disk. The generalization, due to Schneider ([Sch66] or [Nit89], §374), is the following proposition:

Proposition 4.1 (Schneider). Suppose that $D$ is a minimal disk in $\mathbb{R}^{3}$ and that $f: \mathbb{R}^{3} \rightarrow \mathbb{R}$ is a linear function. If $f \mid \partial D$ has at most $n$ local maxima with $f>0$, then $f \mid D$ has at most $(n-1)$ interior critical points, counting multiplicity, with $f \geq 0$.

4.2. A global angle function on $\boldsymbol{H}^{+}$. Let $S$ be the half-strip in the $x z$-plane defined by

$$
S=\{(x, 0, z): x>0,-\pi<z<0\} .
$$

Note that

$$
H^{+}=\bigcup_{\theta \in \mathbb{R}} \sigma_{\theta}(S),
$$

where $\sigma_{\theta}: \mathbb{R}^{3} \rightarrow \mathbb{R}^{3}$ is the screw motion defined by

$$
\sigma_{\theta}(r \cos \phi, r \sin \phi, z)=(r \cos (\phi+\theta), r \sin (\phi+\theta), z+\theta) .
$$

Thus $H^{+}$is foliated by the $\sigma_{\theta}(S)$, and we may think of $\theta$ as a globally defined angle function on $H^{+}$. Furthermore, $\theta$ extends continuously to $\overline{H^{+}} \backslash Z$. Indeed,

$$
\theta: \overline{H^{+}} \backslash Z \rightarrow \mathbb{R}
$$

is the unique continuous function such that

$$
(x, y, z)=\left(\sqrt{x^{2}+y^{2}} \cos \theta, \sqrt{x^{2}+y^{2}} \sin \theta, z\right)
$$

and such that

$$
\theta(x, 0,0)=0 \text { for } x>0 .
$$

Note also that $\theta(x, 0,0)=\pi$ for $x<0$ and that

$$
\theta\left(\sigma_{s}(p)\right)=\theta(p)+s .
$$


It will be useful to understand the helical portions of the boundary curve $\Gamma=$ $\Gamma(R, h)$ (which was defined in Section 3.1) in terms of the angle function $\theta$. By (2) in Section 3.1, there are two such helical portions:

$$
(R \cos v, R \sin v, v), \quad 0 \leq v \leq h
$$

and

$$
(-R \cos v,-R \sin v, v), \quad-h \leq v \leq 0 .
$$

Now

$$
(R \cos v, R \sin v, v)=\sigma_{v}(R, 0,0)
$$

so

$$
\theta(R \cos v, R \sin v, v)=\theta(R, 0,0)+v=v .
$$

Similarly

$$
(-R \cos v,-R \sin v, v)=\sigma_{v}(-R, 0,0)
$$

so

$$
\theta(-R \cos v,-R \sin v, v)=\theta(-R, 0,0)+v=\pi+v .
$$

Hence we can express the upper and lower helical portions of $\Gamma$ in terms of the angle coordinate $\theta$ by substituting $\theta$ for $v$ in (8) and by substituting $\theta-\pi$ for $v$ in (9), yielding

$$
(R \cos \theta, R \sin \theta, \theta), \quad 0 \leq \theta \leq h
$$

for the upper portion and

$$
(R \cos \theta, R \sin \theta, \theta-\pi), \quad-h+\pi \leq \theta \leq \pi
$$

for the lower portion.

The curve $\Gamma$ also contains six line segments: the four horizontal segments joining the endpoints of the helical arcs (10) and (11) to the $z$-axis, and two segments in the $z$-axis (the two segments given by $0<z<h$ and $-h<z<0$ ). See Figure 2 .

\subsection{Vertical tangent planes at points in the interior of $D$}

Proposition 4.4. Let $D$ be a $\rho_{Y}$-invariant minimal embedded disk in $H^{+}$with $\partial D=$ $\Gamma=\Gamma(R, h)$. Let

$$
f(x, y, z)=a x+b y
$$

be a nonzero linear function with $a \leq 0$.

If $a<0$, then

(1) $f \mid D$ has at most one critical point $p$ with $f(p) \geq 0$. Such a critical point must satisfy $0<\theta(p)<2 \pi$. 
(2) $f \mid D$ has at most one critical point $p$ with $f(p) \leq 0$. Such a critical point must satisfy $-\pi<\theta(p)<\pi$.

If $a=0$, then $f \mid D$ has exactly one critical point. That critical point is the unique point of intersection of $D$ and $Y$, and it lies on the positive $y$-axis.

Remark 4.5. There appears to be an asymmetry between assertions (1) and (2), but in fact they are equivalent (either one implies the other). To see this, let $g(x, y, z)=$ $a x-y$. If $p$ is a critical point of $f \mid D$ with $f(p) \leq 0$, then $q=\rho_{Y}(p)$ is a critical point of $g \mid D$ with $g(q) \geq 0$, and $\theta(q)=\pi-\theta(p)$. The apparent asymmetry disappears if the theorem is restated using the angle function $\omega(p):=\theta(p)-\pi / 2$. (Note that $\left.\omega\left(\rho_{Y}(p)\right)=-\omega(p).\right)$

Proof. For $k \in \mathbb{Z}$, let

$$
D_{k}=\{p \in D: 2 \pi k<\theta(p)<2 \pi(k+1)\} .
$$

We will prove that $f \mid D_{k}$ has

(1a) no critical points with $f \geq 0$ if $k \neq 0$, and

(1b) at most one critical point with $f \geq 0$ if $k=0$.

These imply assertion (1), because if $p=(x, y, z) \in D \backslash \cup_{k} D_{k}$, then $\theta(p)$ is an even multiple of $2 \pi$, which implies that $y=0$ and $x>0$ and thus, in the case $a<0$, that $f(p)<0$. (At the end of the proof, we will also use (1a) and (1b) in the case $a=0$.)

Let us first suppose that $h$ is an integer multiple of $2 \pi$ :

$$
h=2 \pi N .
$$

Let $J$ be the halfplane

$$
J=\{(x, 0, z): x \geq 0\}
$$

and let

$$
\Gamma_{k}=\left(\partial D_{k}\right) \backslash J .
$$

Note that if $k \geq N$ or if $k<-N$, then $\Gamma_{k}=\emptyset$, which implies by the convex hull property that $D_{k}=\emptyset$. Thus from now on we assume that $-N \leq k<N$.

By hypothesis, $f \leq 0$ on $J$, so any local maxima of $f \mid \partial D_{k}$ with $f>0$ must lie on $\Gamma_{k}$.

If $k>0$, then $\Gamma_{k}$ consists of the single helical arc:

$$
\{(R \cos \theta, R \sin \theta, \theta): 2 \pi k<\theta<2 \pi(k+1)\} .
$$

Likewise if $k<0$, then $\Gamma_{k}$ consists of the single helical arc

$$
\{(R \cos \theta, R \sin \theta, \theta-\pi): 2 \pi k<\theta<2 \pi(k+1)\} .
$$


(These assertions about $\Gamma_{k}$ follow immediately from (10) and (11) in Section 4.2.) Orthogonal projection to the $x y$-plane maps each of these helical arcs homeomorphically to the circle $x^{2}+y^{2}=R^{2}$ minus the point $(R, 0,0)$. Thus if $k \neq 0$, then $f \mid \partial D_{k}$ has exactly one local maximum with $f>0$. By Schneider's theorem, $f \mid D_{k}$ has no critical points with $f \geq 0$. This proves (1a).

To prove (1b), note that $\Gamma_{0}$ has two connected components. One component is the helical arc

$$
\{(R \cos \theta, R \sin \theta, \theta): 0<\theta<2 \pi\} .
$$

The other connected component of $\Gamma_{0}$ is the helical arc

$$
\{(R \cos \theta, R \sin \theta, \theta-\pi): 0<\theta<\pi\}
$$

together with the line segment

$$
\{(t, 0,0): 0>t>-R\} .
$$

Hence the function $f$ has at most two strictly positive local maxima on $\partial D_{0}$. (If this is not clear, consider the projections to the $x y$-plane as above.) By Schneider's theorem, $f \mid D_{0}$ has at most one critical point with $f \geq 0$. This proves (1b).

This completes the proof of (1a) and (1b) under the hypothesis that $h /(2 \pi)$ is an integer. If $2 \pi(N-1)<h<2 \pi N$, then the topmost component of $\Gamma \backslash J$ is not a full turn of a helix, but rather the helical arc

$$
\{(R \cos \theta, R \sin \theta, \theta): 2 \pi(N-1)<\theta<h\}
$$

together with the line segment

$$
\{(t \cos h, t \sin h, h): 0<t<R\}
$$

Note that this piecewise smooth arc $C$ still has the following property: the function $f(x, y, z)=a x+b y$ has at most one local maximum on $C$ with $f>0$. A similar remark applies to the lowest component of $\Gamma \backslash J$. The rest of the proof of (1a) and of (1b) is exactly as before.

As explained above, (1a) and (1b) imply assertion (1), which in turn (by Remark 4.5) implies assertion (2).

Now suppose that $a=0$. Without loss of generality we may assume that $f(x, y, z)=y$. Since $\rho_{Y}: D \rightarrow D$ is an orientation-preserving involution, it has a unique fixed point $q$. That is, $q$ is the unique point of $D \cap Y$. Since $D \in H^{+}$, the point $q$ lies on the positive $y$-axis. Since $\rho_{Y}: D \rightarrow D$ preserves orientation, so does $\rho_{Y}: \operatorname{Tan}_{q} D \rightarrow \operatorname{Tan}_{q} D$. Thus $\operatorname{Tan}_{q} D$ is perpendicular to $Y$, so $q$ is a critical point of $f \mid D$.

By (1a), $q$ is the only critical point of $f \mid \cup_{k} D_{k}$ with $f \geq 0$. In particular, there is no critical point $p$ of $f \mid D$ for which $\theta(p)$ an odd multiple of $\pi$. By the $\rho_{Y}$ symmetry, 
there is also no critical point $p$ for which $\theta(p)$ an even multiple of $\pi$. Thus $q$ is the only critical point of $f \mid D$ with $f \geq 0$.

Finally, for each $k$ (including $k=0$ ),

$$
\Gamma_{k} \cap\{y<0\}
$$

consists of just one arc (unless it is empty). (This is because $y \geq 0$ on one of the two components of $\Gamma_{0}$.) As before, $f$ has a unique local minimum on each such arc. Thus by Rado's theorem (or by Schneider's theorem applied to the function $-f$ ) $f \mid D$ has no critical points with $f<0$.

Corollary 4.6. Suppose $D \subset H^{+}$is a $\rho_{Y}$-invariant minimal embedded disk with boundary $\Gamma$. Let $V$ be a plane containing the z-axis. If $p \in D$ is a point at which $\operatorname{Tan}_{p} D$ is parallel to $V$, then $p$ lies within distance $2 \pi$ of the plane $z=0$. There are at most two such points. If there are two, they lie on opposite sides of $V$. If there is such a point on $V$, then it satisfies:

$$
0<\theta(p)<\pi
$$

Proof. Note that $V$ can be expressed as the zero set of a nonzero linear function $f(x, y, z)=a x+b y$ with $a \leq 0$, and that $p$ is a critical point of $f \mid D$ if and only if $\operatorname{Tan}_{p} D$ is parallel to $V$. By Proposition 4.4, there are at most two critical points, and if there are two, they must lie on opposite sides of $V$. Also, if $p$ is a critical point, then

$$
-\pi<\theta(p)<2 \pi
$$

which implies that $p$ lies in the slab $|z|<2 \pi$, since, for any $q=(x, y, z) \in H^{+}$

$$
\theta(q)-\pi<z<\theta(q)
$$

Finally, if $p$ is a critical point on $V$, then $f(p)=0$, so combining conclusions (1) and (2) of the theorem gives (12).

Remark 4.7. Proposition 4.4 and Corollary 4.6, and indeed all the results in Section 4, are true for a larger class of curves $\Gamma$. For example, they remain true if $\Gamma$ is obtained from $\Gamma(R, h)$ by replacing the two helical arcs of $\Gamma(R, h)$ with two smooth curves in

$$
\overline{H^{+}} \cap\left\{(x, y, z): x^{2}+y^{2}=R^{2}\right\}
$$

such that the angle function $\theta$ strictly increases from 0 to $h$ on one curve and from $-h+\pi$ to $\pi$ on the other. No changes are required in the proofs. 
4.8. Vertical tangent planes along the axis $Z$. Let $V$ be a vertical plane containing $Z$. Note that $M \cap V$ contains an interval in $Z$. If $M$ and $V$ are tangent at a point $p \in Z$, then $M \cap V$ also contains a curve transverse to $Z$ that passes through $p$. Equivalently, $D \cap V$ contains a curve with $p$ as one of its endpoints. Analyzing the curves of intersection of $D$ and $V$ will allow us to understand the distribution of points on $Z$ where $V$ and $M$ are tangent. This analysis is carried out in Theorem 4.10.

We begin with a simple lemma.

Lemma 4.9. The intersection of $\bar{D}$ with any plane in $\mathbb{R}^{3}$ cannot contain a closed curve unless the curve passes through the origin.

Proof. Consider a closed curve $C$ in $\bar{D}$ not passing through the origin. Since $\bar{D} \backslash\{0\}$ is an embedded disk, $C$ bounds a portion $D^{\prime}$ of $D$. If a plane contained $C$, then by the maximum principle that plane would contain $D^{\prime}$, and therefore by analytic continuation it would contain all of $D$, which is impossible since $\Gamma$ is not a planar curve.

Theorem 4.10. Let $V$ be a plane that contains $Z$. Let $W$ be a connected component of $\mathrm{H}^{+} \cap V$ that intersects $D$ transversely and that does not contain any horizontal segment of $\Gamma(R, h)$ in its boundary. Then $W \cap D$ consists of at most three curves, and at most four of the endpoints of those curves are on $Z$.

Proof. (See Figure 5.) Note that $W=\sigma_{\theta}(S)$ for some $\theta \in \mathbb{R}$, where $S$ is the halfstrip

$$
\{(x, 0, z): x>0,-\pi<z<0\}
$$

as in Section 4.2. Equivalently,

$$
W=\left\{p \in H^{+}: \theta(p)=\theta\right\} .
$$

By transversality, $D \cap W$ consists of smooth curves.

By Lemma 4.9, none of the curves in $D \cap W$ is closed. By elementary topology, the endpoints of such curves must lie in the set

$$
(\partial D) \cap \bar{W}=I \cup P
$$

where $I$ is an interval in the $z$-axis, namely

$$
\{(0,0, z): z \in[-h, h] \cap[\theta-\pi, \theta]\}
$$

and where $P$ is the intersection of the helical portion of $\Gamma$ with $\bar{W}$.

Note that $P$ consists of zero, one, or both (depending on $\theta$ ) of the points

$$
a(\theta)=(R \cos \theta, R \sin \theta, \theta)
$$




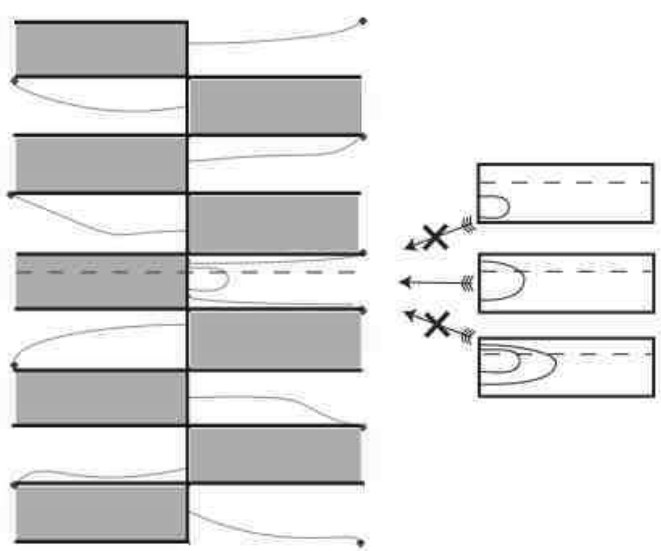

Figure 5. The possible intersections of a vertical plane $V$ and an embedded minimal disk $D$, where $Z \subset V, D \subset H^{+}$, and $\partial D=\Gamma(R, h)$. The dotted horizontal line is at the level $z=0$. The straight line segments represent $H \cap V$. The unshaded rectangles lie in $H^{+} \cap V$, the shaded ones in $H^{-} \cap V$. The curves in $H^{+} \cap V$ indicate possible intersections of $V$ and $D$. The component of $H^{+} \cap V$ that contains points at the level of $z=0$ is the only component that can contain more than one intersection curve, and the only component in which an intersection curve might conceivably have both endpoints on $Z$.

and

$$
b(\theta)=(R \cos \theta, R \sin \theta, \theta-\pi) .
$$

(See (10) and (11) in Section 4.2.) Also note that each element of $P$ is indeed the endpoint of exactly one curve of $D \cap W$. Thus at most two curves in $D \cap W$ can have an endpoint in $P$. These curves together have at most two endpoints on the $z$-axis. Any other curve $C$ of $D \cap W$ must have both endpoints on the $z$-axis. Note one endpoint of $C$ must be on the positive $z$-axis and the other on the negative $z$ axis, since otherwise $C$ together with the segment joining its endpoints would violate Lemma 4.9. Furthermore, there cannot be a second curve $C^{\prime}$ from the positive $z$-axis to the negative $z$-axis, since $C \cup C^{\prime}$ together with the two segments joining their endpoints (and not containing the origin) would then violate Lemma 4.9.

If we assume that $\bar{W}$ is disjoint from the plane $z=0$, then the method of proof of Theorem 4.10 gives considerably more:

Proposition 4.11. Suppose $W=\sigma_{\theta}(S)=\left\{p \in H^{+}: \theta(p)=\theta\right\}$.

(1) If $\bar{W}$ is disjoint from the plane $z=0$, then $D$ intersects $W$ transversely.

(2) If $\bar{W}$ is contained in the interior of the slab

$$
K=\{(x, y, z):|z| \leq \max \{h, \pi\}\},
$$


and is disjoint from the plane $z=0$, then $D \cap W$ consists of a single smooth embedded curve with exactly one endpoint on $Z$.

(3) If $\bar{W}$ is not contained in the slab $K$, then $D \cap W$ is empty.

(4) If $\bar{W}$ is contained in the slab $2 \pi<z<h$ or in the slab $-h<z<-2 \pi$, then $D \cap W$ is a single smooth embedded curve with one endpoint on $Z$, and that curve is a graph over a line segment in the plane $z=0$.

Proof. The hypothesis in assertion (1) is equivalent to the condition that $\theta$ is not in the interval $[0, \pi]$. The transversality thus follows from Corollary 4.6.

In assertion (2), the set $P$ in the proof of Theorem 4.10 consists of a singe point, and that proof then shows that $D \cap W$ is a single curve with exactly one endpoint on $Z$.

In assertion (3), the set $P$ is empty, from which it follows that $D \cap W$ is also empty.

In assertion (4), $D \cap W$ consists of a single smooth curve by assertion (2). The hypothesis implies that $\theta$ is not contained in the interval $[-\pi, 2 \pi]$. By Theorem 4.4, that curve has no vertical tangents and thus is a graph over its projection to the plane $z=0$.

4.12. An upper bound on the number of tangent planes parallel to a vertical plane $\boldsymbol{V}$. Let $D$ be a $\rho_{Y}$-invariant minimal embedded disk in $H^{+}$with boundary $\Gamma=\Gamma(R, h)$, let

$$
M=\bar{D} \cup \rho_{Z}(\bar{D}),
$$

and let $N$ be the corresponding $\sigma_{2 h}$-invariant surface. We combine the results of the two previous sections to get a local bound on the number of points of $N$ at which that the tangent plane is parallel to a given vertical plane. The estimate is independent of $R$ and $h$.

Theorem 4.13. Let $V$ be a plane containing $Z$, and let $\Pi$ be an open horizontal slab of thickness $\pi$ :

$$
\Pi=\{(x, y, z): a<z<a+\pi\} .
$$

Then $\Pi$ contains at most sixteen points of $N$ at which the tangent plane is parallel to $V$.

The number sixteen is certainly not optimal, but for our purposes any finite number would suffice.

Proof. Consider first a plane $V$ containing $Z$ that is generic in the following sense: $V$ intersects $N \backslash Z$ transversely and $V$ does not contain any of the countably many horizontal line segments in $N$. Now $N$ is made up of congruent copies of $M$, which 
is in turn made up of two copies of $\bar{D}$. Note that $\Pi$ contains points from at most two copies of $M$, and therefore from at most four copies of $\bar{D}$. Now $\operatorname{Tan}_{p} N$ is not vertical at any boundary point of $N$ by the boundary maximum principle. Also, $V$ does not contain any of the horizontal edges of the copies of $\bar{D}$. Thus if $\operatorname{Tan}_{p} N$ is parallel to $V$, then $p$ lies either on the $z$-axis or else in the interior of one of the four copies of $\bar{D}$. By Theorem 4.10 (see also the discussion in 4.8), there are at four such points on $Z$ in each copy of $M$. By Corollary 4.6, there are at most two such points not on $Z$ in each copy of $D$. Thus there are at most $2 \times 4+4 \times 2$ or sixteen such points in $\Pi$.

By openness of the Gauss map, the number of such points is a lower semicontinuous function of $V$. Thus the bound for arbitrary $V$ follows from the bound for generic $V$.

\section{Uniform estimates}

5.1. A uniform curvature estimate. Consider a $\rho_{Y}$-invariant minimal embedded disk $D$ in $H^{+}$with boundary $\partial D=\Gamma=\Gamma(R, h)$, the curve specified in Section 3.1. Extend $D$ by Schwarz reflection in $Z$ to get a minimal embedded genus-one surface $M$ with $\partial M=\partial H_{R, h}$. As observed in Section 2.1, this boundary consists of a top line segment, a bottom line segment, and two helical arcs. By repeated Schwarz reflection in the top and bottom line segments, we get a smooth embedded minimal surface $N$ invariant under the screw motion $\sigma_{2} h$. The boundary of $N$ consists of two helices.

In Section 6, we will obtain complete nonperiodic (or periodic) genus-one helicoids by letting $R$ and $h$ (or just $R$ ) tend to infinity. In this section, we prove the estimates that allow us to control passage to the limit.

Lemma 5.2. Suppose $D$ is a $\rho_{Y}$-invariant minimal embedded disk in $H^{+}$with boundary $\partial D=\Gamma=\Gamma(R, h)$. Let

$$
M=\bar{D} \cup \rho_{Z}(\bar{D})
$$

and let $N$ be the $\sigma_{2 h}$-invariant surface obtained from $M$.

(1) If $C$ is a closed curve in $N$ that does not intersect any straight line or straight line segment contained in $N$, then $C$ is contractible in $N$.

(2) Any pair of disjoint homologically nontrivial embedded closed curves in M must bound an annulus in $M$.

(3) There is a unique shortest homotopically nontrivial curve $\alpha$ in $\bar{D}$. It is a smooth closed geodesic in $N$ that is bisected by 0 together with the unique point of $Y \cap D$. 
Proof. Note that if we remove the straight line segments from $N$, we are left with a disjoint union of pieces, each of which is congruent to $D$ and therefore is simply connected. In assertion (1), $C$ lies entirely in one of those pieces and is therefore contractible in it.

In Section 2.2, we showed that $M$ is topologically a once-punctured torus. Assertion (2) follows by standard, elementary topology.

Let $\alpha$ be a shortest curve that is a generator for $\pi_{1}(\bar{D}, 0) \cong \mathbb{Z}$. (The fundamental group is infinite cyclic since $\bar{D}$ is topologically a closed disk with two boundary points identified.) Note that $N$ has geodesically convex boundary because the curvature vector at each point of its bounding helices points toward the $z$-axis. Since $\bar{D}$ is bounded by portions of $\partial N$ together with geodesics in $N$, the curve $\alpha$ does not touch $\partial D$ except at 0 . Thus $\alpha$ is a smoothly embedded geodesic in $\bar{D}$ that starts and ends at 0 . Since $D$ has nonpositive curvature, $\alpha$ is a unique. Therefore it is $\rho_{Y}$ invariant, so its midpoint $p$ is a fixed point of $\rho_{Y}$, namely the unique point of $Y \cap D$. (See Proposition 4.4.) The $\rho_{Y}$ invariance also implies that the two components of $\alpha \backslash\{0, p\}$ are related by $\rho_{Y}$. This implies that $\alpha$ does not have a corner at 0 , but rather forms a smooth closed geodesic in $N$.

Remark 5.3. Note that conclusions (1) and (2) of Lemma 5.2 are preserved under smooth convergence. That is, if $M_{i}$ and $N_{i}$ are smooth minimal surfaces satisfying the first two conclusions of the lemma, and if the $M_{i}$ and $N_{i}$ converge smoothly to limits $M$ and $N$, then $M$ and $N$ also satisfy those two conclusions.

The proofs of our next two results rely strongly on the following theorem of Mo and Osserman [MO90], which extends earlier work of Osserman [Oss63], Xavier [Xav81], Sa Earp-Rosenberg [SER88], and Fujimoto [Fuj88]. (The Sa Earp-Rosenberg result is also strong enough for our purposes.)

Theorem 5.4 (Mo-Osserman). If $\mathcal{N}$ is a complete minimal surface in $\mathbb{R}^{3}$ with Gauss map $g: \mathcal{N} \rightarrow \mathbb{S}^{2}$ and if the set

$$
\left\{v \in \mathbb{S}^{2}: g^{-1}(v) \text { is finite }\right\}
$$

contains five or more points, then $\mathcal{N}$ has finite total curvature.

We now give our main curvature estimate:

Theorem 5.5. There are finite constants $R_{0}$ and $K$ with the following property. Suppose $D$ is an $\rho_{Y}$-invariant minimal embedded disk in $H^{+}$with boundary $\Gamma(R, h)$, where $R \geq R_{0}$ and $h \geq \pi / 2$. Then

$$
B(D, p) \leq K .
$$


Here $B(D, p)$ is the norm of the second fundamental form of $D$ at $p$.

The hypothesis $h \geq \pi / 2$ could be removed, since one can show that there is no such disk $D$ if $h<\pi / 2$. However, we only require the theorem for $h \geq \pi / 2$.

Proof. Suppose the theorem is false. Then there is a sequence of examples $D_{i}$ with $\partial D_{i}=\Gamma\left(R_{i}, h_{i}\right)$ and a sequence of points $p_{i} \in \bar{D}_{i}$ such that $B\left(D_{i}, p_{i}\right)$ and $R_{i}$ tend to infinity and such that $h_{i} \geq \pi / 2$.

Let

$$
M_{i}=\overline{D_{i}} \cup \rho_{Z}\left(\overline{D_{i}}\right),
$$

let $N_{i}$ be the screw-motion-invariant surface obtained from $M_{i}$, and let $C_{i}$ be the solid cylinder of radius $R_{i}$ about the $z$-axis.

We may suppose that $p_{i}$ has been chosen in $\overline{D_{i}}$ to maximize $B\left(\overline{D_{i}}, p_{i}\right)=$ $B\left(N_{i}, p_{i}\right)$. (The maximum exists because $N_{i}$ is smooth and $\bar{D}_{i}$ is a compact subset of $N_{i}$.) It follows that

$$
\max _{p \in N_{i}} B\left(N_{i}, p\right)=B\left(N_{i}, p_{i}\right) .
$$

Translate $N_{i}, M_{i}$, and $C_{i}$ by $-p_{i}$, and then dilate by $B\left(N_{i}, p_{i}\right)$ to get $N_{i}^{\prime}, M_{i}^{\prime}$, and $C_{i}^{\prime}$. By passing to subsequences we may assume that $N_{i}^{\prime}, M_{i}^{\prime}$, and $C_{i}^{\prime}$ converge as sets to limits $N^{\prime}, M^{\prime}$, and $C^{\prime}$.

Note that

$$
\max _{p \in N_{i}^{\prime}} B\left(N_{i}^{\prime}, p\right)=B\left(N_{i}^{\prime}, 0\right)=1 .
$$

Also, either $\partial N_{i}^{\prime}$ converges to the empty set or else it converges smoothly to a horizontal line. (There is just one line since $h_{i} \geq \pi / 2$ and since the dilation factor $B\left(D_{i}, p_{i}\right)$ tends to infinity. The line is horizontal since $R_{i} \rightarrow \infty$.) This together with (13) implies that the convergence $N_{i}^{\prime} \rightarrow N^{\prime}$ is smooth. Thus the limit is a smooth, embedded minimal surface and $\partial N^{\prime}$ is either the empty set or a horizontal line.

Let

$$
\mathcal{N}= \begin{cases}N^{\prime} & \text { if } \partial N^{\prime}=\emptyset, \\ N^{\prime} \cup \rho_{\partial N^{\prime}}\left(N^{\prime}\right) & \text { if } \partial N^{\prime} \neq \emptyset .\end{cases}
$$

If $\partial N^{\prime}$ is a line, then $N^{\prime}$ lies in a halfspace (namely $C^{\prime}$ ) whose boundary plane contains that line, and thus $\mathcal{N}$ is embedded. Of course if $\partial N^{\prime}$ is empty, then $\mathcal{N}$ is also embedded. Either way, $\mathcal{N}$ is a complete embedded minimal surface.

By Theorem 4.13, $N^{\prime}$ has the following property: if $V$ is a vertical plane, then there are at most a finite number of points of $N^{\prime}$ at which the tangent plane is parallel to $V$. (If this is not clear, recall that the $h_{i}$ are bounded below and that the dilation factor $B\left(D_{i}, p_{i}\right)$ tends to infinity.) Note that if $L$ is a horizontal line, then $\rho_{L}\left(N^{\prime}\right)$ has the same property. Consequently $\mathcal{N}$ also has this property. In other words, the set

$$
\left\{v \in \mathbb{S}^{2}: g^{-1}(v) \text { is finite }\right\}
$$


contains a great circle, where $g: \mathcal{N} \rightarrow \mathbb{S}^{2}$ is the Gauss map. Hence by the MoOsserman theorem 5.4, $\mathcal{N}$ has finite total curvature.

We now know that $\mathcal{N}$ is a complete embedded minimal surface of finite total curvature. By (13),

$$
B\left(\mathcal{N}, p^{\prime}\right)=1,
$$

so $\mathcal{N}$ is not flat. Therefore $\mathcal{N}$ has a catenoidal end. Intersecting the end with a suitable plane parallel to the end, we see that $\mathcal{N}$ contains a planar closed curve that does not intersect any straight line segment contained in $\mathcal{N}$. By assertion (1) of Lemma 5.2, that curve bounds a disk in $\mathcal{N}$. By the maximum principle, the disk must lie in the plane containing its boundary. But then by analyticity, all of $\mathcal{N}$ must be planar, contradicting (14).

The following theorem will let us conclude that the complete surfaces we construct are nearly horizontal away from the $z$-axis.

Theorem 5.6. Suppose $D_{i}$ is a sequence of embedded, $\rho_{Y}$-invariant minimal disks in $H^{+}$with $\partial D_{i}=\Gamma\left(R_{i}, h_{i}\right)$, where $R_{i} \rightarrow \infty$ and $h_{i} \rightarrow h \in(\pi / 2, \infty]$. Let $D_{i}^{\prime}$ be the result of translating $D_{i}$ by $-p_{i}$, where $p_{i} \in D_{i}$ is a sequence of points such that

$$
\operatorname{dist}\left(p_{i}, Z \cup \partial C_{i}\right) \rightarrow \infty .
$$

Here $\operatorname{dist}\left(p_{i}, \cdot\right)$ denotes intrinsic distance in $D_{i}$ and $C_{i}$ denotes the solid cylinder of radius $R_{i}$ about $Z$.

After passing to a subsequence, the $D_{i}^{\prime}$ converge smoothly to a limit $D^{\prime}$. Let $\Sigma$ be the component of $D^{\prime}$ containing 0 . Then

(1) $\Sigma$ is a horizontal plane, or

(2) $\Sigma$ is a horizontal halfplane, or

(3) $\partial \Sigma$ consists of two lines parallel to the $x$-axis.

The third case can occur only if $h=\pi$, the intrinsic $\operatorname{dist}\left(p_{i}, X\right)$ from $p_{i}$ to $X$ is bounded, and the length of the shortest closed geodesic in $\overline{D_{i}}$ tends to infinity.

Remark 5.7. One can prove (using a slight generalization of Proposition 5.13 below) that in case (3), the surface $\Sigma$ must be a flat strip. However, we will not need that fact. Our main construction (Section 6) uses disks with the annular intersection property, and Theorem 5.11 below implies that case (3) does not occur for disks $D_{i}$ having that property.

Proof. By the convex hull property, $D_{i}$ lies in the solid cylinder $C_{i}$. By Theorem 5.5, we may assume (after passing to a subsequence) that the $D_{i}^{\prime}$ converge smoothly to $D^{\prime}$ and that the corresponding translates of the $C_{i}$ and of $H$ converge to a limits $C^{\prime}$ and $H^{\prime}$, respectively. 
Note that $H^{\prime}$ is either a helicoid or else a union of horizontal planes according to whether the Euclidean distance from $p_{i}$ to $Z$ stays bounded or tends to infinity. Similarly, since $R_{i} \rightarrow \infty$, the limit $C^{\prime}$ is either a halfspace bounded by a vertical plane or else all of $\mathbb{R}^{3}$ according to whether the Euclidean distance from $p_{i}$ to $\partial C_{i}$ stays bounded or tends to infinity.

By Corollary 4.6, for each vertical plane $V$, there are at most two points of $\Sigma$ at which the tangent plane is parallel to $V$.

Consider first the case $\partial \Sigma=\emptyset$. By the Mo-Osserman Theorem 5.4, $\Sigma$ has finite total curvature. Also, it is complete, embedded, and simply connected. Therefore it is a plane.

Since $H$ and $D_{i}$ are disjoint, $H^{\prime}$ and $\Sigma$ cannot cross each other (i.e., contain points of transverse intersection). Now $H^{\prime}$ is either a helicoid or a union of horizontal planes. Since the plane $\Sigma$ crosses every helicoid, $H^{\prime}$ must be a union of parallel planes. Since $\Sigma$ does not cross $H^{\prime}$, the plane $\Sigma$ must also be horizontal.

Note that the horizontal plane $\Sigma$ is contained in $C^{\prime}$, so $C^{\prime}$ must be all of $\mathbb{R}^{3}$ (rather than a halfspace bounded by a vertical plane.) Since $H^{\prime}$ is a union of horizontal planes and $C^{\prime}$ is all of $\mathbb{R}^{3}$, it follows, as explained above, that the Euclidean distance from $p_{i}$ to $Z \cup \partial C_{i}$ tends to infinity in the case $\partial \Sigma=\emptyset$.

Now suppose $\partial \Sigma$ is not empty. By (15), the boundary $\partial \Sigma$ consists of one or more horizontal lines corresponding to the horizontal radial segments in $\partial D_{i}$. Since intrinsic distance in $\overline{D_{i}}$ and Euclidean distance coincide on line segments in $\overline{D_{i}} \backslash\{0\}$, the hypothesis (15) implies that the Euclidean distance from $p_{i}$ to $Z \cup \partial C_{i}$ tends to infinity. This in turn implies that $H^{\prime}$ is a union of horizontal planes.

Suppose that $\partial \Sigma$ consists of a single horizontal line $L$. Let

$$
\Sigma^{*}=\Sigma \cup L \cup \rho_{L} \Sigma .
$$

Then (just as before) $\Sigma^{*}$ is a complete, embedded, simply connected minimal surface of finite total curvature. Thus $\Sigma^{*}$ is a plane, so $\Sigma$ is a half-plane. Since $D_{i}$ lies in $H^{+}, \Sigma$ must lie in the closed region between two successive planes in $H^{+}$. Thus $\Sigma$ is horizontal.

Finally, suppose $\partial \Sigma$ consists of more than one horizontal line. Since $D_{i}$ lies in $H^{+}, \Sigma$ lies in the region between two successive planes in $H^{\prime}$. That is, $\Sigma$ lies in a horizontal slab $\Omega$ of thickness $\pi$. It follows that $\partial \Sigma$ consists of exactly two horizontal lines, one in each component of $\partial \Omega$. The vertical distance between those two lines is $\pi$.

On the other hand, the horizontal segments in $\partial D_{i}=\Gamma\left(R_{i}, h_{i}\right)$ lie in the planes $z=-h_{i}, z=0$, and $z=h_{i}$. Thus the vertical distance between two lines in $\partial \Sigma$ is either $h$ or $2 h$. Thus $h=\pi$ or $2 h=\pi$. Since $h>\pi / 2$, this means $h=\pi$.

Since $h_{i} \rightarrow \pi$, the horizontal edges of $\partial D_{i}$ converge to the positive and negative portions of the $x$-axis and to the rays $\{(x, 0, \pi): x \leq 0\}$ and $\{(x, 0,-\pi): x \geq 0\}$. 
Thus the two lines of $\partial \Sigma$ are parallel, and (after passing to a subsequence if necessary) they are limits either:

(1) of $X^{-}$and of the top horizontal edge $T_{i}$, both translated by $-p_{i}$, or

(2) of $X^{+}$and the bottom horizontal edge $B_{i}$, both translated by $-p_{i}$.

Of course in either case $\operatorname{dist}\left(p_{i}, X\right)$ must be bounded. Note in case (1), $\operatorname{dist}\left(p_{i}, T_{i}\right)$ is also bounded, and in case (2) $\operatorname{dist}\left(p_{i}, B_{i}\right)$ is bounded.

Without loss of generality, assume we are in case (1), so that $\operatorname{dist}\left(p_{i}, X^{-}\right)$and $\operatorname{dist}\left(p_{i}, T_{i}\right)$ are bounded.

Let $\alpha_{i}$ be the shortest closed curve in $\overline{D_{i}}$. Then $\alpha_{i}$ divides $\Gamma\left(R_{i}, h_{i}\right)$ into two components. One component consists of the top edge $T_{i}$, the edge in positive $x$-axis, and a helical arc joining them. The other component consists of the bottom edge $B_{i}$, the edge in the negative $x$-axis, and a helical arc joining them. In particular, the top horizontal edge and the edge in the negative $X$-axis belong to different components of $\Gamma\left(R_{i}, h_{i}\right) \backslash \alpha_{i}$ and thus in different components of $D_{i} \backslash \alpha_{i}$. Hence either the shortest curve from $p_{i}$ to $T_{i}$ or the shortest curve from $p_{i}$ to $X^{-}$must cross $\alpha_{i}$. Therefore the union of those two curves with $\alpha_{i}$ contains a path joining $p_{i}$ to the origin. Thus

$$
\operatorname{dist}\left(p_{i}, 0\right) \leq \operatorname{Length}\left(\alpha_{i}\right)+\operatorname{dist}\left(p_{i}, T_{i}\right)+\operatorname{dist}\left(p_{i}, X^{-}\right) .
$$

Since the left hand side tends to infinity and since the second and third terms on the right are bounded, Length $\left(\alpha_{i}\right)$ must tend to infinity.

In proving the theorem, we also proved

Corollary 5.8. If the intrinsic distance from $p_{i}$ to $Z \cup \partial C_{i}$ tends to infinity, then the Euclidean distance from $p_{i}$ to $Z \cup \partial C_{i}$ also tends to infinity.

The following special case of Theorem 5.6 will be used in 5.11:

Corollary 5.9. If $\operatorname{dist}\left(p_{i}, X \cup Z \cup \partial C_{i}\right) \rightarrow \infty$, then $\operatorname{Tan}_{p_{i}} D_{i}$ converges to a horizontal plane.

Proof. Since $\operatorname{dist}\left(p_{i}, Z \cup \partial C_{i}\right) \rightarrow \infty$, assertion (1), (2), or (3) of Theorem 5.6 must hold. Since $\operatorname{dist}\left(p_{i}, X\right) \rightarrow \infty$, assertion (3) does not hold.

5.10. A uniform bound on the length of the closed geodesic in $\bar{D}$. Consider a $\rho_{Y}$-invariant, minimal embedded disk $D$ in $H^{+}$with boundary $\Gamma(R, h)$ (for some $R$ and $h)$, where $\Gamma(R, h)$ is the curve defined in Section 3.1. The next theorem establishes a uniform estimate for the length of the shortest closed geodesic in $\bar{D}$, provided the disk $D$ has the annular intersection property 3.2. (This is the only estimate in the paper that depends on the annular intersection property.) We will use this result to show that the genus-one surfaces $M=\bar{D} \cup \rho_{Z}(\bar{D})$ have genus-one limits as $R \rightarrow \infty$. 
Theorem 5.11. Suppose $D_{i}$ is a sequence of $\rho_{Y}$-invariant minimal embedded disks in $\mathrm{H}^{+}$with boundary $\Gamma\left(R_{i}, h_{i}\right)$ and with the annular intersection property. Suppose also that

$$
h_{i} \geq \eta>\pi / 2
$$

and that $R_{i} \rightarrow \infty$. Then the length of the shortest closed geodesic in $\overline{D_{i}}$ is bounded above.

Proof. Let $\alpha_{i}$ be the shortest closed geodesic in $\overline{D_{i}}$. By assertion (3) of Lemma 5.2, $\alpha_{i}$ contains both the origin and the unique point $p_{i}$ in $D_{i} \cap Y$, and the length of $\alpha_{i}$ is twice the intrinsic distance from $p_{i}$ to 0 . Thus it suffices to bound $\operatorname{dist}\left(p_{i}, 0\right)$ above.

Let $q_{i}$ be the point in $X \cup Z$ that is closest in intrinsic distance to $p_{i}$. Then

$$
\begin{aligned}
\operatorname{dist}\left(p_{i}, 0\right) & \leq \operatorname{dist}\left(p_{i}, q_{i}\right)+\operatorname{dist}\left(q_{i}, 0\right) \\
& =\operatorname{dist}\left(p_{i}, q_{i}\right)+\left|q_{i}\right| \\
& \leq \operatorname{dist}\left(p_{i}, q_{i}\right)+\left|p_{i}-q_{i}\right| \\
& \leq 2 \operatorname{dist}\left(p_{i}, q_{i}\right) .
\end{aligned}
$$

(Here $\left|q_{i}\right| \leq\left|p_{i}-q_{i}\right|$ because $p_{i} \in Y$ and $q_{i} \in X \cup Z$ are orthogonal.) Thus

$$
\operatorname{dist}\left(p_{i}, 0\right) \leq 2 \operatorname{dist}\left(p_{i}, X \cup Z\right) .
$$

The tangent plane to $D_{i}$ at $p_{i}$ is vertical by Proposition 4.4. Thus by Corollary 5.9, the sequence

$$
\operatorname{dist}\left(p_{i}, X \cup Z \cup \partial C_{i}\right)
$$

is bounded, which implies by (16) that the sequence

$$
\operatorname{dist}\left(p_{i},\{0\} \cup \partial C_{i}\right)
$$

is bounded. Hence it suffices to prove that

$$
\operatorname{dist}\left(p_{i}, \partial C_{i}\right) \rightarrow \infty
$$

Suppose, on the contrary, that $\operatorname{dist}\left(p_{i}, \partial C_{i}\right)$ is bounded. Translate $D_{i}, H$, and $C_{i}$ by $-p_{i}$ to get $D_{i}^{\prime}, H_{i}^{\prime}$, and $C_{i}^{\prime}$. Since $\operatorname{dist}\left(p_{i}, \partial C_{i}\right)$ is bounded and $R_{i} \rightarrow \infty$, the $C_{i}^{\prime}$ converge (after passing to a subsequence) to a halfspace $C^{\prime}$ of the form

$$
C^{\prime}=\{(x, y, z): y \leq a\}
$$

It follows that the $H_{i}^{\prime}$ converge smoothly to a limit $H^{\prime}$ consisting of the horizontal planes on which $z$ is an odd multiple of $\pi / 2$. By the curvature estimate in Theorem 5.5, we may assume that the $D_{i}^{\prime}$ converge smoothly to a limit surface. Let $D^{\prime}$ be the 
connected component of that limit surface containing the origin. Then $D^{\prime}$ lies in one of the components of $C^{\prime} \backslash H^{\prime}$, namely the halfslab

$$
\{(x, y, z): y \leq a \text { and }|z|<\pi / 2\} .
$$

Note that $\partial D^{\prime}$ consists of the two straight line edges of the halfslab. (By the $\rho_{Y}$ symmetry of $D^{\prime}$, the boundary $\partial D^{\prime}$ cannot be just one of the two lines.)

By Corollary $5.8, \bar{D}^{\prime}$ is properly embedded.

We have shown that $\bar{D}^{\prime}$ is a simply connected properly embedded minimal surface in the half-slab (18) and that $\partial D^{\prime}$ consists of the two edges of (18). The only such minimal surface is the vertical strip $S$ bounded by those two edges (see Proposition 5.13 below), so $D^{\prime}=S$. However, we will show that $D^{\prime}=S$ contradicts the annular intersection property of the $D_{i}$.

Let $A^{\prime}$ be a catenoid that intersects the planes $z= \pm \pi / 2$ in a pair of circles in the region $y<a$. Since $D_{i}$ intersects each annulus in $\mathcal{A}\left(\Gamma_{i}\right)$, it follows that $D^{\prime}$ intersects $A^{\prime}$. But the strip $D^{\prime}=S$ does not intersect $A^{\prime}$, so we have a contradiction.

(In case it is not clear, we spell out in more detail why $D^{\prime}$ must intersect $A^{\prime}$. Let $A_{i}^{\prime}$ be the component of $A^{\prime} \backslash H_{i}^{\prime}$ that crosses the $x y$-plane. Translate $A_{i}^{\prime}$ by $p_{i}$ to get $A_{i}$. Because of the smooth convergence, when $i$ is sufficiently large, $A_{i}$ will be in $\mathcal{A}\left(\Gamma_{i}\right)$, and so $D_{i}$ will intersect $A_{i}$. Thus $D_{i}^{\prime}$ intersects $A_{i}^{\prime}$ and hence, passing to the limit, $D^{\prime}$ intersects $A^{\prime}$.)

Remark 5.12. Note that the annular intersection property was only used to prove (17). Thus, even without assuming the annular intersection property, the theorem applies to any sequence $D_{i}$ for which (17) holds.

Proposition 5.13. Suppose $U \subset \mathbb{R}^{3}$ is a half-slab bounded by two horizontal halfplanes and an infinite strip $S$. Suppose $M$ is a simply connected, properly embedded minimal surface in $\bar{U}$ with $\partial M=\partial S$. Then $M=S$.

Proof. We may suppose that $U=\{(x, y, z): y<0$ and $|z|<h\}$ and thus that the strip is $S=\{(x, y, z): y=0$ and $|z|<h\}$.

Since $M$ is connected, it contains an embedded path $\gamma$ joining $(0,0,-h)$ to $(0,0, h)$. Since $M$ is simply connected, $\gamma$ divides $M$ into two components. One of those components, which we will denote $M^{+}$, has boundary consisting of $\gamma$ together with $\{(x, 0, \pm h) \in \partial S: x \geq 0\}$.

(If this is not clear, note that if $M \neq S$, then $M \cup S$ bounds a region in $\mathbb{R}^{3}$ and so is orientable. Thus $M \cup S$ is topologically an annulus (rather than a Möbius strip), and thus $\gamma$ together with the segment joining its endpoints divides $M \cup S$ into two components.)

Let $\mathbb{B}$ be a ball centered at the origin and containing $\gamma$. Let $\mathcal{C}$ be the set of complete catenoids $C$ with vertical axes of symmetry such that $C$ is disjoint from 
$\mathbb{B} \cup\{(x, y, z) \in S: x \geq 0\}$. Note that $\mathcal{C}$ is a connected family, and that there are catenoids in $\mathcal{C}$ that are disjoint from $M^{+}$. (Take any $C \in \mathcal{C}$ disjoint from $\bar{U}$.) Thus by the maximum principle, all the catenoids in $\mathcal{C}$ are disjoint from $M^{+}$. These catenoidal barriers force $M^{+}$to be arbitrarily close to $S$ near $\infty$ :

$$
\lim _{(x, y, z) \in M^{+}, x \rightarrow \infty} y=0 .
$$

Likewise for $(x, y, z) \in M^{-}=M \backslash M^{+}$, we see that $y \rightarrow 0$ as $x \rightarrow-\infty$. Thus $|y|$ achieves its maximum on $M$ at a finite point $p$. By the maximum principle, this maximum value must be 0 .

\section{The main theorems}

Theorem 6.1. There exists a complete, properly embedded minimal surface $M$ in $\mathbb{R}^{3}$ such that

(1) $M \cap H=X \cup Z$.

(2) Each of the two components of $M \backslash H$ is simply connected.

(3) $M$ is topologically a once-punctured torus.

(4) $\operatorname{Tan}_{p} M$ converges to a horizontal plane as $\operatorname{dist}(p, Z) \rightarrow \infty$.

(5) The points of $M \backslash Z$ with vertical tangent planes lie in a cylinder $\mathbb{B}(0, R) \times$ $[-2 \pi, 2 \pi]$.

(6) $M$ is conformally a once-punctured torus.

(7) The level set $M \cap\{z=0\}$ consists of $X$ together with a smooth, simple closed curve that intersects $X$ in exactly two points.

(8) For each $c \neq 0$, the level set $M \cap\{z=c\}$ consists of a single smooth, nonclosed curve.

(9) $M$ is asymptotic to $H$ at infinity.

Proof. Choose sequences $R_{i}$ and $h_{i}$ tending to infinity. By Propositions 3.3 and 3.11, for all sufficiently large $i$, the curve $\Gamma\left(R_{i}, h_{i}\right)$ bounds a $\rho_{Y}$-invariant minimal embedded disk $D_{i}$ in $H^{+}$with the annular intersection property. Let

$$
M_{i}=\overline{D_{i}} \cup \rho_{Z}\left(\overline{D_{i}}\right) .
$$

By Theorem 5.5, the curvatures of the $M_{i}$ are uniformly bounded, so (by passing to a subsequence) we may assume that the $M_{i}$ converge to a complete, embedded minimal surface $M$. 
Now

$$
\partial\left(M_{i} \cap H^{+}\right)=\partial D_{i}=\Gamma\left(R_{i}, h_{i}\right) \rightarrow X \cup Z .
$$

It follows that

$$
\partial\left(M \cap H^{+}\right)=X \cup Z
$$

which implies assertion (1).

Since $M \cap H^{+}$is the limit of the simply connected minimal surfaces $M_{i} \cap H^{+}=$ $D_{i}$, it must also be simply connected. Similarly $M \cap H^{-}$must be simply connected. This proves assertion (2).

Furthermore, $M$ is proper by Corollary 5.8 .

By assertion (3) of Lemma 5.2, $M_{i}$ contains a simple closed geodesic $\alpha_{i}$ such that $0 \in \alpha_{i}$ and such that $\alpha_{i} \backslash\{0\}$ lies in $D_{i}$. Thus $\alpha_{i}^{\prime}=\rho_{Z}\left(\alpha_{i}\right)$ is another closed geodesic, and $\alpha_{i}$ and $\alpha_{i}^{\prime}$ intersect transversely at the origin and nowhere else. (They intersect only at the origin because $\alpha_{i} \backslash\{0\}$ lies in $D_{i} \subset H^{+}$and $\alpha_{i}^{\prime} \backslash\{0\}$ lies in $\rho_{Z}\left(D_{i}\right) \subset H^{-}$.)

The lengths of the geodesics $\alpha_{i}$ and $\alpha_{i}^{\prime}$ are bounded above by Theorem 5.11, and they are bounded below since the curvatures of the $M_{i}$ are uniformly bounded (Theorem 5.5). Thus (after passing to a subsequence if necessary) the $\alpha_{i}$ and $\alpha_{i}^{\prime}$ converge to closed geodesics $\alpha$ and $\alpha^{\prime}$ in $M$ that intersect transversely at the origin. Thus $M$ has genus at least one. By part (2) of Lemma 5.2 (see also Remark 5.3), $M$ has genus at most one. Thus $M$ has genus exactly one. Assertion (2) of Lemma 5.2 also implies that $M$ has exactly one end. Thus $M$ is topologically a once-punctured torus.

Assertion (4) follows from Theorem 5.6.

Assertion (5) follows from assertion (4) together with Corollary 4.6.

To prove the remaining assertions, it is convenient first to prove the following:

Claim. Let $M^{+}=M \cap H^{+}$.

(i) If $c \geq 0$, then $M^{+} \cap\{z>c\}$ has exactly one connected component.

(ii) If $\operatorname{Tan}_{p} M$ is horizontal, then $p \in X \backslash\{0\}$.

(iii) $M^{+} \cap\{z=0\}$ consists of a single smooth embedded curve.

(iv) Either $M \cap\{z=0\}$ consists of three connected components each of which is a smooth embedded curve, or it consists of $X$ together with a smooth, simple closed curve that crosses $X$ exactly twice.

Proof of claim. To prove (i), let $C$ be the component of $M^{+} \cap\{z>c\}$ that contains

$$
\{(0,0, z): z>c\}
$$

in its boundary, and let

$$
C^{\prime}=\left(M^{+} \cap\{z>c\}\right) \backslash C
$$


If $p \in M^{+} \cap\{z>c\}$ and if $\theta(p)>c+2 \pi$ (where $\theta$ is the angle function defined in Section 4.2), then by assertion (4) of Proposition 4.11, there is a curve in $M^{+} \cap\{z>c\}$ that contains $p$ and that has an endpoint on $Z$. Thus such a point $p$ must lie in the component $C$. This shows that the function $\theta$ is bounded above on $C^{\prime}$. Thus the function $z$ is also bounded above on $C^{\prime}$. (Recall that $\theta(x, y, z)$ and $z$ differ by at $\operatorname{most} \pi$.)

Now $C^{\prime}$ is a minimal surface that is properly embedded in $\{z>c\}, C^{\prime}$ is contained in a slab, and $\partial C^{\prime} \subset\{z=c\}$. A version of the halfspace theorem [HM90] states that if a connected, properly immersed minimal surface $\Sigma$ lies in a slab, and $\partial \Sigma$ (if nonempty) lies on one boundary face of the slab, then $\Sigma$ is a subset of a plane. Hence $C^{\prime}$ is a union of horizontal planes. But $C^{\prime}$ is contained in $H^{+}$, so $C^{\prime}$ must be empty. This proves (i).

To prove (ii), suppose the horizontal plane $\{z=c\}$ is tangent to $M$ at some point $p$. By the $\rho_{Y}$ symmetry, we may assume without loss of generality that $c \geq 0$. If $p$ were in $M^{+}$, then by a theorem of Rado ([Rad71], III.7 or [Oss86], Lemma 7.5), the tangent plane $\{z=c\}$ would divide $M^{+}$into four of more components, at least two of which would lie in the region $z>c$, contradicting (i). Thus $p$ does not lie in $M^{+}$. By the same argument (or by $\rho_{Z}$ symmetry), it also cannot lie in $M^{-}$, the other component of $M \backslash H$. Thus by assertion (1), $p \in X \cup Z$. Since $Z \subset M$, the tangent plane to $M$ at every point of $Z$ is vertical. Thus $p \in X \backslash\{0\}$. This proves (ii).

To prove (iii), note that if $M^{+} \cap\{z=0\}$ contained more than a single embedded curve, then $M^{+} \backslash\{z=0\}$ would have more than two components. By the $\rho_{Y}$ symmetry, it would have more than one component in the halfspace $\{z>0\}$. But to according to (i) (with $c=0$ ), there is only one such component.

To prove (iv), let $S$ be the curve whose existence is given by statement (iii) of the claim. If $\bar{S}$ has no endpoints, then it follows from statement (iii) that $M \cap\{z=0\}$ consists of the three components $X, S$, and $\rho_{X}(S)$, each of which is a smooth, embedded curve. Now suppose that $\bar{S}$ has an endpoint $p$. Then $p$ must be a singular point of $M \cap\{z=0\}$. Thus the tangent plane to $M$ at $p$ must be horizontal. By statement (ii) of the claim, the point $p$ must lie on $X \backslash\{0\}$. By the $\rho_{Y}$-symmetry of $M$, the point $-p$ must also be an endpoint of $\bar{S}$. Thus $S$ consists of $X$ together with the simple closed curve $\bar{S} \cup \rho_{X}(S)$, which intersects $X$ precisely at the two points $p$ and $-p$. This completes the proof of the claim.

We have established that $M$ is a complete, properly immersed minimal surface with finite topology, one end, and bounded curvature, such that the level set

$$
M \cap\{z=0\}
$$

consists of finitely many curves with finitely many singular points (points where curves cross). According to a theorem of Rodriguez and Rosenberg [RR98], any minimal surface $M$ with these properties has finite type, meaning: 
(a) $M$ is conformally a once-punctured Riemann surface. Equivalently, the onepoint compactification $M \cap\{\infty\}$ is conformally a compact Riemann surface (in our case a torus).

(b) The one-form $(d g) / g$ (where $g$ is the Gauss map) is meromorphic on $M \cup\{\infty\}$,

(c) Let $\eta$ be the holomorphic one-form on $M$ whose real part is $d v$, where $v(x, y, z)=z$ is the height function. Then $\eta$ is a meromorphic one-form on $M \cup\{\infty\}$.

In particular, (a) is assertion (6) of the theorem.

Since the height function $v$ is harmonic and nonconstant on $M$, the one-form $\eta$ must have a pole at $\infty$ and nowhere else. Since $\eta$ is a nonconstant meromorphic one-form on a torus, it must have an equal number (counting multiplicity) of zeroes and poles. Thus it must have some zeroes on $M$. In other words, the height function $v$ must have critical points on $M$. By statement (ii) of the claim, those critical points must lie on $X \backslash\{0\}$. In particular, the level set $M \cap\{z=0\}$ is not everywhere smooth. Thus by statement (iv) of the claim, the level set consists of $X$ together with a simple closed curve that intersects $X$ at two points. This is assertion (7).

By elementary complex analysis, for each $c \in \mathbb{R}$, the number of ends of the level set $M \cap\{z=c\}$ (i.e., of $v^{-1}(c)$ ) is equal to the order of the pole of $\eta$ at $\infty$. For $c=0$, there are two ends by assertion (7). Thus $\eta$ has a double pole at $\infty$, and the level set $M \cap\{z=c\}$ has two ends at infinity for every $c$.

Now let $c \neq 0$. Since the height function has no critical points at height $c$, the level set

$$
M^{+} \cap\{z=c\}
$$

is a union of smooth embedded curves. None of the curves is closed since $M^{+}$is simply connected and embedded. Thus the level set $M \cap\{z=c\}$ (which is obtained from (19) by reflection in $Z$ ) is a disjoint union of non-closed smooth curves. We have just shown that this level set has exactly two ends. Thus it consists of exactly one curve. This proves assertion (8).

It remains only to show assertion (9), that $M$ is asymptotic to $H$ at infinity. This follows from immediately from a theorem of Hauswirth, Perez, and Romon, who prove that any embedded minimal surface of finite type, one end, bounded curvature, and infinite total curvature must be asymptotic to a helicoid at infinity [HPR01].

The Hauswirth-Perez-Romon Theorem is very general, but has a rather lengthy proof. We can also deduce assertion (9) from the following theorem (due to Hoffman and McCuan), which has a much shorter proof.

Theorem ([HM03]). Let $E \subset \mathbb{R}^{3}$ be a properly immersed, minimal annular end that is conformally a punctured disk. Suppose that $\frac{d g}{g}$ and $\eta$ both have double poles at the puncture and that $\eta$ has no residue at the puncture. If E contains a vertical ray and a horizontal ray, then a sub-end of $E$ is embedded and asymptotic to a helicoid. 
We have already proved that the end of $M$ satisfies all the hypotheses except for two: that $\eta$ has no residue at $\infty$, and that $d g / g$ has a double pole at $\infty$. (We showed in the proving assertion (8) that $\eta$ has a double pole at $\infty$.)

Since $\eta$ has no poles on $M$, it has no residue at $\infty$ by Stokes' theorem.

Since $d g / g$ and $\eta$ are meromorphic one-forms on $M \cup\{\infty\}$, their ratio is a meromorphic function and hence has a limiting value at value at infinity:

$$
\lim _{p \rightarrow \infty} \frac{d g / g}{\eta}(p)=\xi \in \mathbb{C} \cup\{\infty\} .
$$

Recall ([HK97], p. 15) that the principal curvatures at a point are $\pm \kappa$ where

$$
\kappa=4\left(|g|+\frac{1}{|g|}\right)^{-2}\left|\frac{d g / g}{\eta}\right| .
$$

On $Z$, the tangent plane is vertical, so $|g|=1$ and

$$
\kappa=\left|\frac{d g / g}{\eta}\right| .
$$

Thus by (20),

$$
\lim _{p \rightarrow \infty, p \in Z} \kappa(p)=|\xi| .
$$

Since $M$ has bounded principal curvatures, $\xi \neq \infty$.

Note that on any interval $I \subset \mathbb{Z}^{+}$of length $4 \pi$, the tangent plane to $H$ turns through angle $4 \pi$. Since $M^{+}$lies on one side of $H$, this forces the tangent plane to $M$ to turn through an angle at least $3 \pi$ on $I$. Thus there must be a point in $I$ at which

$$
\kappa \geq \frac{3 \pi}{4 \pi}=\frac{3}{4} .
$$

In particular, there is a sequence of such points in $Z$ tending to $\infty$, so $\xi \neq 0$ by (21).

Since $\xi$ is finite and nonzero, (20) implies that $d g / g$ and $\eta$ have poles of the same order at $\infty$. Since $\eta$ has a double pole at $\infty$, so does $d g / g$.

All the hypotheses of the Hoffman-McCuan Theorem are satisfied, so $M$ is asymptotic to $H$. This completes the proof of Theorem 6.1.

Theorem 6.2. For each $h>\pi / 2$, there is a complete, properly embedded minimal surface $N=N(h)$ in $\mathbb{R}^{3}$ such that

(1) The intersection $N \cap H$ of $N$ with the helicoid $H$ consists of the z-axis together with the horizontal lines

$$
H \cap\{z=n h\}, \quad n \in \mathbb{Z} .
$$

Furthermore, $N \backslash H$ consists of congruent, simply connected components. 
(2) $N$ is invariant under the screw motion $\sigma_{2 h}$.

(3) The portion $M$ of $N$ in the slab $|z|<h$ is bounded by the two horizontal lines $\rho_{ \pm h}(X)$.

(4) The quotient $N / \sigma_{2 h}$ has finite total curvature and is conformally a twice-punctured torus.

(5) $\operatorname{Tan}_{p} N$ converges to a horizontal plane as $\operatorname{dist}(p, Z) \rightarrow \infty$.

(6) The quotient $\mathrm{N} / \sigma_{2 h}$ is asymptotic to $\mathrm{H} / \sigma_{2 h}$ at infinity.

Proof. Fix an $h>\pi / 2$ and choose a sequence $R_{i} \rightarrow \infty$. By Propositions 3.3 and 3.11, for sufficiently large $i$ there is a $\rho_{Y}$-invariant minimal embedded disk $D_{i}$ in $H^{+}$with boundary $\Gamma\left(R_{i}, h\right)$ and with the annular intersection property. Let

$$
M_{i}=\overline{D_{i}} \cup \rho_{Z}\left(\overline{D_{i}}\right)
$$

and let $N_{i}$ be the $\sigma_{2 h}$-invariant surface obtained from $M_{i}$ by repeated Schwarz reflections. (Or, equivalently, let $N_{i}=\cup_{n \in \mathbb{Z}} \sigma_{2 h n} M_{i}$.)

By Theorem 5.5, the curvatures of the $N_{i}$ are uniformly bounded, so (passing to a subsequence) we may assume that the $N_{i}$ converge smoothly to a complete embedded $\sigma_{2 h}$-invariant minimal surface $N$. It follows that the $M_{i}$ converge smoothly to the closure of the surface

$$
M=\{(x, y, z) \in N:|z|<h\} .
$$

Note that $N$ is orientable since it is properly embedded in $\mathbb{R}^{3}$. If $L$ is a line in $N$, then the rotation $\rho_{L}: N \rightarrow N$ is orientation-reversing. Thus

$$
\sigma_{2 h}: N \rightarrow N
$$

is orientation-preserving since it is the product of two such rotations (corresponding to $L=X$ and $L=H \cap\{z=h\})$. Therefore $N / \sigma_{2 h}$ is orientable.

Assertions (1), (2), and (3) follow immediately from the construction.

Exactly as in the proof of Theorem 6.1, $M$ is homeomorphic to $T \backslash \Delta$ where $T$ is a torus and $\Delta$ is a closed disk in $T$. Since $\bar{M}$ is $M$ together with its two boundary lines, $\bar{M}$ is homeomorphic to the union of $T \backslash \Delta$ with two disjoint arcs of $\partial \Delta$. If we identify those two arcs, either the result is non-orientable or else it is topologically a twice punctured torus. Since $N / \sigma_{2 h}$ is orientable and since it is the result of identifying the two boundary lines of $\bar{M}$, it follows that $N / \sigma_{2 h}$ is topologically a twice-punctured torus.

Note that the total curvatures of the $M_{i}$ are uniformly bounded by the GaussBonnet theorem. It follows that $N / \sigma_{2 h}$ has finite total curvature, which implies by Huber's theorem [Hub57] (or by [Oss86], Theorem 9.1)) that it is conformally a punctured Riemann surface. Thus it is a conformally a twice-punctured torus. 
Assertion (5), i.e., that $\operatorname{Tan}_{p} N$ becomes horizontal as $\operatorname{dist}(p, Z) \rightarrow \infty$, follows from Theorem 5.6. (Note that case (3) of that theorem does not arise because Theorem 5.11 gives a uniform bound on the lengths of the closed geodesics in the $\overline{D_{i}}$.)

We now show that the two horizontal ends are helicoidal rather than planar. (See [MR93] for a discussion of the possible end behavior of embedded periodic minimal surfaces.) If they were planar, one end would correspond to the plane $z=0$ and the other to the plane $z=h$ (since $N$ contains horizontal lines at those heights). But $\rho_{Y}$ preserves orientation on $N$ and reverses orientation on the plane $z=0$. Thus the ends cannot be planar. (Section 5 of $[\mathrm{HW}]$ gives another proof that the ends of $N / \sigma_{2 h}$ are asymptotic to a helicoid.)

It remains only to show that $D$ is asymptotic to $H$ as $x^{2}+y^{2} \rightarrow \infty$ rather than to some other helicoid.

Recall that $\overline{D_{i}}$ has a unique embedded, closed geodesic. Note that the geodesic divides $\overline{D_{i}}$ into two components, and that the portions of $X^{+}$and of $\sigma_{h}\left(X^{+}\right)$in $\bar{D}_{i} \backslash\{0\}$ belong to the same connected component. (They are joined by a helical arc in $\overline{D_{i}}$ ). Thus by smooth convergence of $D_{i} \rightarrow D$ and of the corresponding geodesics, we see that the simple closed geodesic in $\bar{D}$ divides it into two components, one of which, $D^{\text {upper }}$, contains $X^{+}$and $\sigma_{h} X^{+}$. Consequently $X^{-}$and $\sigma_{-h}\left(X^{-}\right)$belong to the other component.

Let $R$ be large enough that the closed geodesic and all points of $\bar{D}$ with vertical tangent planes lie in the open cylinder of radius $R$ about $Z$. Then for each $r \geq R$,

$$
\overline{D^{\text {upper }}} \cap\left\{x^{2}+y^{2}=r^{2}\right\}
$$

is a smooth compact curve, on each component of which the angle function

$$
\theta: H^{+} \rightarrow \mathbb{R}
$$

is monotonic. Thus no component is a closed curve, and since there are exactly two endpoints, namely $(r, 0,0)$ and $\sigma_{h}(r, 0,0)$, there is exactly one component. Furthermore, that component can be parametrized by $\theta$ and therefore written as:

$$
(r \cos \theta, r \sin \theta, f(r, \theta)) \quad(\alpha \leq \theta \leq \beta)
$$

for suitable $\alpha$ and $\beta$. Now $\theta=0$ on $X^{+}$and $\theta=h$ on $\sigma_{h}\left(X^{+}\right)$, so $\alpha=0, \beta=h$, $f(r, 0)=0$, and $f(r, h)=h$.

Since this is true for all $r \geq R$, we have proved that outside of the cylinder of radius $R$ about $Z, \overline{D^{\text {upper }}}$ may be written as

$$
\{(r \cos \theta, r \sin \theta, f(r, \theta)): r \geq R, 0 \leq \theta \leq h\}
$$

where

$$
f(r, 0)=0, \quad f(r, h)=h .
$$


Since $D$ is asymptotic to a helicoid, $\partial f / \partial \theta$ converges to a constant as $r \rightarrow \infty$, namely the pitch of the helicoid. Thus (22) implies that the constant is 1 and that

$$
f(r, \theta)=\theta+o(r)
$$

which implies that $D^{\text {upper }}$ is asymptotic to $H$.

\section{A compactness theorem}

Let $\mathcal{N}$ be the class of all symmetric, embedded genus-one helicoids, periodic and nonperiodic. If $N \in \mathcal{N}$ is periodic, let $h(N)$ be the smallest $h>0$ such that $N$ is $\sigma_{2 h}$-invariant. If $N$ is nonperiodic, let $h(N)=\infty$.

For each $h>\pi / 2$, we have proved existence of an $N \in \mathcal{N}$ with $h(N)=h$. Furthermore, there are no $N \in \mathcal{N}$ with $h(N) \leq \pi / 2$ (see [HW], Section 3). However, $\mathcal{N}$ may conceivably contain examples that do not arise from our construction. Nevertheless, the following two theorems apply to all symmetric, embedded genus-one helicoids.

Theorem 7.1. If $\eta>\pi / 2$, then the class

$$
\mathcal{N}_{\eta}:=\{N \in \mathcal{N}: h(N) \geq \eta\}
$$

is compact with respect to smooth convergence on compact sets of $\mathbb{R}^{3}$. Furthermore, each surface $N \in \mathcal{N}$ has all the properties listed in Theorem 6.1 if $h(N)=\infty$, or in Theorem 6.2 if $h(N)<\infty$.

Proof. If $R=\infty$ and/or $h=\infty$, let us (by a slight abuse of notation) interpret the expression " $\partial D=\Gamma(R, h)$ " to mean " $\partial D=\partial Q_{R, h}$ and $D$ is asymptotic to $Q_{R, h}$ at infinity". Note that if $R=\infty$, then $\partial Q_{R, h}$ has no helical portions, but rather consists entirely of horizontal rays together vertical segments or rays. In another paper ([HW], Section 2), we will prove that if $N \in \mathcal{N}$, then

$$
D:=\left\{(x, y, z) \in N \cap H^{+}:|z|<h(N)\right\}
$$

is a disk with $\partial D=\Gamma(\infty, h(N))$.

We claim that all of the results in Sections 4 and 5 of this paper remain true for any disk $D$ with $\partial D=\Gamma(R, h)$, even when $R$ and/or $h$ is allowed to be infinite. By Remark 4.7, Propositions 4.4 and 4.10 are true for $D \cap C$ for any sufficiently large solid cylinder $C$ about $Z$, and thus those propositions are also true for $D$. No changes are required in any of the other proofs. (In particular, one works directly with $D$ rather than with the portion of $D$ in a solid cylinder.) By Remark 5.12, Theorem 5.11 is true when $R_{i} \equiv \infty$ even without assuming the annular intersection property. 
Consider a sequence $N_{i} \in \mathcal{N}_{\eta}$. By the discussion above, the $N_{i}$ and the disks

$$
D_{i}:=\left\{(x, y, z) \in N_{i} \cap H^{+}:|z|<h\left(N_{i}\right)\right\}
$$

satisfy all the estimates in Sections 4 and 5. In particular, the curvature estimates imply that a subsequence of the $N_{i}$ converges to a limit $N$, and Theorem 5.11 implies that the sequence of points $D_{i} \cap Y$ is bounded. The proofs of Theorem 6.1 and Theorem 6.2 then show that $N$ satisfies all the conclusions of Theorem 6.1 (if $h(N)=\infty$ ) or Theorem 6.2 (if $h(N)<\infty$ ), and so in particular $N \in \mathcal{N}_{\eta}$.

We end by pointing out that our bounds on points with vertical tangent planes are uniform:

Theorem 7.2. For every $\eta>\pi / 2$, there is an $R<\infty$ with the following property. If $N \in \mathcal{N}_{\eta}$ and if $p$ is a point in the fundamental domain

$$
M=\{(x, y, z) \in N:|z|<h(N)\}
$$

such that $\operatorname{Tan}_{p} N$ is vertical, then $p$ lies on the z-axis or in the solid cylinder

$$
\left\{(x, y, z): x^{2}+y^{2} \leq R^{2},|z|<2 \pi\right\} .
$$

Proof. This follows immediately from Propositions 5.6 and 5.11.

\section{Appendix. Existence of unstable minimal disks}

Here we prove the minimax principle used in Section 3.

Let $U$ be an open subset of $\mathbb{R}^{3}$. We will call $U$ mean convex provided no smooth minimal surface in $\bar{U}$ whose boundary is in $U$ can touch $\partial U$. We will call $U$ strictly mean convex provided no smooth minimal surface $M$ in $\bar{U}$ can touch $\partial U$ except at its boundary $\partial M$. For example, in case that $\partial U$ is smooth, $U$ is mean convex if and only if the mean curvature of $\partial U$ (with respect to the inward-pointing normal) is everywhere nonnegative, and $U$ is strictly mean convex if and only if the mean curvature is positive on dense subset of $\partial U$.

Suppose $U$ has piecewise smooth boundary. If each face has nonnegative mean curvature with respect to the inward-pointing normal, and if the interior angle along each edge is less than $\pi$, then $U$ is mean convex.

Theorem A.1. Suppose $\Gamma$ is a smooth, simple closed curve in $\mathbb{R}^{3}$ and that $D_{1}$ and $D_{2}$ are two disjoint strictly stable smooth embedded minimal disks in $\mathbb{R}^{3}$ with $\partial D_{1}=$ $\partial D_{2}=\Gamma$. Suppose also that $D_{1}$ and $D_{2}$ meet transversely along $\Gamma$. Then $\Gamma$ bounds $a$ weakly unstable, smoothly embedded minimal disk in the region between $D_{1}$ and $D_{2}$. 
Proof. Let $U$ be the region between $D_{1}$ and $D_{2}$. Enlarge $U$ slightly by pushing each $D_{i}$ outward by $\epsilon u_{i}$ times the outward unit normal, where $u_{i}$ is the first eigenfunction of the Jacobi operator on $D_{i}$, normalized to have maximum value 1. By strict stability, the resulting enlarged domain $U_{\epsilon}$ will be strictly mean convex for all sufficiently small $\epsilon>0$. (See Proposition A.3 below.) Since the first eigenfunction is positive everywhere, $U_{\epsilon}$ contains $U$ provided $\epsilon>0$.

Now we appeal to the following theorem:

Theorem A.2. Suppose that $W$ is a mean convex region in $\mathbb{R}^{3}$ and that $V$ is a smooth open subset of $\partial W$. Then:

(1) A generic, smooth, simple closed curve $C$ in $V$ is non-critical in that 0 is not an eigenvalue of the Jacobi operator of any smooth embedded disk in $W$ with boundary $C$.

(2) If $W$ is strictly mean convex and if $C$ is a non-critical smooth simple closed curve in $V$ that bounds a disk in $V$, then $C$ bounds finitely many smooth embedded minimal disks in $W$, and the number of even-index disks is exactly one more than the number of odd-index disks.

This theorem is stated for strictly convex $W$ with smooth boundary in Theorem 2.1 of [Whi89]. But the proof given there, which is inspired by Tomi-Tromba [TT78], actually establishes the more general result stated here.

To continue the proof of Theorem A.1, let $\Gamma^{\prime}$ be such a generic curve in either of the two smooth faces of $U_{\epsilon}$. By strict stability of $D_{i}(i=1,2)$ and by the implicit function theorem (cf. Theorem 3.1 of [Whi87a]), if $\Gamma^{\prime}$ is sufficiently close to $\Gamma$, then $\Gamma^{\prime}$ will bound a smooth embedded minimal disk $D_{i}^{\prime}$ that is a slight perturbation of $D_{i}$. In particular, $\Gamma^{\prime}$ bounds at least two strictly stable disks in $U_{\epsilon}$. Thus by Theorem A.2, it must bound at least one minimal embedded disk $D^{\prime}$ in $U_{\epsilon}$ that has odd index and that is therefore strictly unstable.

Now take a subsequential limit of such $D^{\prime}$ for a sequence of $\Gamma^{\prime}$ s converging to $\Gamma$. The resulting surface is a weakly unstable disk $D(\epsilon)$ in $U_{\epsilon}$. Now let $D$ be a subsequential limit of the $D(\epsilon)$ as $\epsilon \rightarrow 0$. Then $D$ is a weakly unstable smooth embedded minimal disk in $\bar{U}$. It is not equal to $D_{1}$ or to $D_{2}$ since they are both strictly stable. Hence by the strict maximum principle, $D$ cannot touch $D_{1}$ or $D_{2}$. That is, $D \subset U$.

Proposition A.3. Let $D$ be a smooth, embedded, strictly stable, orientable minimal surface in $\mathbb{R}^{3}$. Let $v$ be a unit normal vectorfield on D. Suppose $u$ is an eigenfunction corresponding to the first eigenvalue of the Jacobi operator on $D$. Then for all sufficiently small $t \neq 0$, the surface $D(t)$ parameterized by $x \in D \mapsto x+t u(x) v(x)$ lies on one side of $D$ and has nonzero mean curvature vector that points into the region between $D$ and $D(t)$. 
Proof. Let $j$ be a positive integer and define an operator $\Phi: C^{j+2}(\bar{D}) \rightarrow C^{j}(\bar{D})$ on a neighborhood of the zero function as follows. Given $w \in C^{j+2}(\bar{D})$, form the disk $x \mapsto x+w(x) v(x)$. Let $\vec{h}(w)(x)$ be the mean curvature vector of this surface at the point $x+w(x) v(x)$, and let

$$
\Phi(w)(x)=\vec{h}(x) \cdot v(x) .
$$

Then $\Phi$ is a smooth map of Banach spaces. Also, $\Phi(0)=0$ and $\Phi^{\prime}(0)$ is the Jacobi operator $J$. Thus if $w$ is any smooth function on $\bar{D}$, then

$$
\Phi(w)=J(w)+O\left(|w|^{2}\right) .
$$

In particular, letting $w=t u$ gives

$$
\Phi(t u)=-t \lambda u+O\left(t^{2}\right)
$$

where $\lambda$ is the first eigenvalue of $J$. By strict stability, $\lambda>0$. Thus

$$
\frac{\Phi(t u)}{t} \rightarrow-\lambda u \text { in } C^{j} \quad \text { as } t \rightarrow 0 .
$$

Now $u$ does not vanish on the interior of $D$, and hence by the boundary maximum principle $D u$ never vanishes at the boundary. Thus the $C^{j}$ convergence (23) implies (for all sufficiently small $t \neq 0$ ) that $\Phi(t u)$ never vanishes on the interior of $D$ and that it has the sign indicated in the statement of the lemma.

Corollary A.4. Suppose D is a strictly stable, embedded orientable minimal surface in $\mathbb{R}^{3}$. Then there is an open set $W$ containing $D$ with the following property. If $M$ is a minimal surface in $W$ with $\partial M \subset D$, then $M \subset D$.

Proof. Choose $\delta>0$ so that for $|t| \leq \delta$, the disk $D(t)$ has the property asserted in Proposition A.3. Let

$$
W=\bigcup_{|t|<\delta} D(t)
$$

Given a minimal surface $M$ in $W$ with $\partial M \subset D$, let $T$ be the largest number such that $M \cap D_{T}$ is nonempty. Then $T=0$, since if $T$ were positive, the maximum principle would be violated at the point of contact of $M$ and $D_{T}$. Likewise the smallest $T$ such that $\bar{M} \cap D_{T}$ is nonempty is 0 .

Remark A.5. Using the boundary maximum principle, one can show that the corollary is also true for $M \subset W$ with $\partial M \subset \bar{D}$. 


\section{References}

[CHM89] M. Callahan, D. Hoffman, and W. H. Meeks, III, Embedded minimal surfaces with an infinite number of ends. Invent. Math. 96 (1989), 459-505. Zbl 0676.53004 MR 0996552

[CM04a] T. H. Colding and W. P. Minicozzi, II, The space of embedded minimal surfaces of fixed genus in a 3-manifold. I. Estimates off the axis for disks. Ann. of Math. (2) 160 (1) (2004), 27-68. Zbl 1070.53031 MR 2119717

[CM04b] T. H. Colding and W. P. Minicozzi, II, The space of embedded minimal surfaces of fixed genus in a 3-manifold. II. Multi-valued graphs in disks. Ann. of Math. (2) $\mathbf{1 6 0}$ (1) (2004), 69-92. Zbl 1070.53032 MR 2119718

[CM04c] T. H. Colding and W. P. Minicozzi, II, The space of embedded minimal surfaces of fixed genus in a 3-manifold. III. Planar domains. Ann. of Math. (2) 160 (2) (2004), 523-572. Zbl 1076.53068 MR MR2123932

[CM04d] T. H. Colding and W. P. Minicozzi, II, The space of embedded minimal surfaces of fixed genus in a 3-manifold. IV. Locally simply connected. Ann. of Math. (2) $\mathbf{1 6 0}$ (2) (2004), 573-615. Zbl 1076.53069 MR 2123933

[FM05] L. Ferrer and F. Martín, Minimal surfaces with helicoidal ends. Math. Z. 250 (4) (2005), 807-839. Zbl 02235449 MR 2180376

[Fuj88] H. Fujimoto, On the number of exceptional values of the Gauss maps of minimal surfaces. J. Math. Soc. Japan 40 (2) (1988), 235-247. Zbl 0629.53011 MR 0930599

[HPR01] L. Hauswirth, J. Pérez, and P. Romon, Embedded minimal ends of finite type. Trans. Amer. Math. Soc. 353 (4) (2001), 1335-1370. Zbl 0986.53005 MR 1806738

[HK97] D. Hoffman and H. Karcher, Complete embedded minimal surfaces of finite total curvature. In Geometry, V, Encyclopaedia Math. Sci. 90, Springer-Verlag, Berlin 1997, 5-93, 267-272. Zbl 0890.53001 MR 1490038

[HKW93] D. Hoffman, H. Karcher, andF. Wei, The genus one helicoid and the minimal surfaces that led to its discovery. In Global Analysis and Modern Mathematics (ed. by K. Uhlenbeck), Publish or Perish Press, 1993. 119-170. Zbl 1049.53502 MR 1278754

[HKW99] D. Hoffman, H. Karcher, and F. Wei, The singly periodic genus-one helicoid. Comment. Math. Helv. 74 (1999), 248-279. Zbl 0958.53006 MR 1691949

[HM03] D. Hoffman and J. McCuan, Embedded minimal annular ends asymptotic to the helicoid. Comm. Anal. Geom. 11 (4) (2003), 721-735.Zbl 1079.53016 MR 2015173

[HM88] D. Hoffman and W. H. Meeks, III, A variational approach to the existence of complete embedded minimal surfaces. Duke Math. J. 57 (3) (1988), 877-893. Zbl 0676.53006 MR 0975126

[HM90] D. Hoffman and W. H. Meeks, III, The strong halfspace theorem for minimal surfaces. Invent. Math. 101 (2) (1990), 373-377. Zbl 0722.53054 MR 1062966

[HW02] D. Hoffman and F. Wei, Deforming the periodic genus-one helicoid. Experiment. Math. 11 (2) (2002), 207-218. Zbl 1116.53301 MR 1959264

[HW] D. Hoffman and B. White, The geometry of genus-one helicoids. To appear in Comment. Math. Helv. 
[Hub57] A. Huber, On subharmonic functions and differential geometry in the large. Comment. Math. Helv. 32 (1957), 13-72. Zbl 0080.15001 MR 0094452

[MR93] W. H. Meeks, III and H. Rosenberg, The geometry of periodic minimal surfaces. Comment. Math. Helv. 68 (4) (1993), 538-578. Zbl 0807.53049 MR 1241472

[MR05] W. H. Meeks, III and H. Rosenberg, The uniqueness of the helicoid. Ann. of Math. 161 (2005), 727-758. Zbl 02201328 MR 2153399

[MY82a] W. H. Meeks, III and S. T. Yau, The classical Plateau problem and the topology of three-dimensional manifolds. Topology 21 (4) (1982), 409-442. Zbl 0489.57002 MR 0670745

[MY82b] W. H. Meeks, III and S. T. Yau, The existence of embedded minimal surfaces and the problem of uniqueness. Math. Z. 179 (1982), 151-168. Zbl 0479.49026 MR 0645492

[MO90] X. Mo and R. Osserman, On the Gauss map and total curvature of complete minimal surfaces and an extension of Fujimoto's theorem. J. Differential Geom. 31 (2) (1990), 343-355. Zbl 0666.53003 MR 1037404

[Nit89] J. C. C. Nitsche, Lectures on Minimal Surfaces. Volume 1. Cambridge University Press, Cambridge 1989. Zbl 0688.53001 MR 1015936

[Oss63] R. Osserman, On complete minimal surfaces. Arch. Rational Mech. Anal. 13 (1963), 392-404. Zbl 0127.38003 MR 0151907

[Oss86] R. Osserman, A Survey of Minimal Surfaces. 2nd edition, Dover Publications, New York 1986. MR 0852409

[Rad71] T. Radó, On the problem of Plateau. Subharmonic functions. Reprint, SpringerVerlag, New York 1971. Zbl 0211.13803 MR 0344979

[RR98] L. Rodríguez and H. Rosenberg, Minimal surfaces in $\mathbf{R}^{3}$ with one end and bounded curvature. Manuscripta Math. 96 (1) (1998), 3-7. Zbl 0968.53009 MR 1624411

[SER88] R. Sa Earp and H. Rosenberg, On values of the Gauss map of complete minimal surfaces in $\mathbf{R}^{3}$. Comment. Math. Helv. 63 (1988), 579-586. Zbl 0694.53007 MR 0966950

[Sch66] R. Schneider, A note on branch points of minimal surfaces. Proc. Amer. Math. Soc. 17 (1966), 1254-7. Zbl 0152.38804 MR 0202068

[TT78] F. Tomi and A. J. Tromba, Extreme curves bound an embedded minimal surface of disk type. Math. Z. 158 (1978), 137-145. Zbl 0353.53032 MR 0486522

[WHW06] M. Weber, D. Hoffman, and M. Wolf, An embedded genus-one helicoid. To appear in Ann. of Math..

[Whi87a] B. White, The space of $m$-dimensional surfaces that are stationary for a parametric elliptic functional. Indiana Univ. Math. J. 36 (3) (1987), 567-602. Zbl 0770.58005 MR 0905611

[Whi87b] B. White, Curvature estimates and compactness theorems in 3-manifolds for surfaces that are stationary for parametric elliptic functionals. Invent. Math. 88 (2) (1987), 243-256. Zbl 0615.53044 MR 0880951

[Whi89] B. White, New applications of mapping degrees to minimal surface theory. J. Differential Geom. 29 (1989), 143-162. Zbl 0638.58005 MR 0978083 
[Xav81] F. Xavier, The Gauss map of a complete non-flat minimal surface cannot omit 7 points of the sphere. Ann. of Math. 113 (1981), 211-214. Zbl 0477.53007 MR 0657243

Received October 22, 2006

David Hoffman, Department of Mathematics, Stanford University, Stanford, CA 94305,

U.S.A.

E-mail: hoffman@math.stanford.edu

Brian White, Department of Mathematics, Stanford University, Stanford, CA 94305, U.S.A.

E-mail: white@math.stanford.edu 